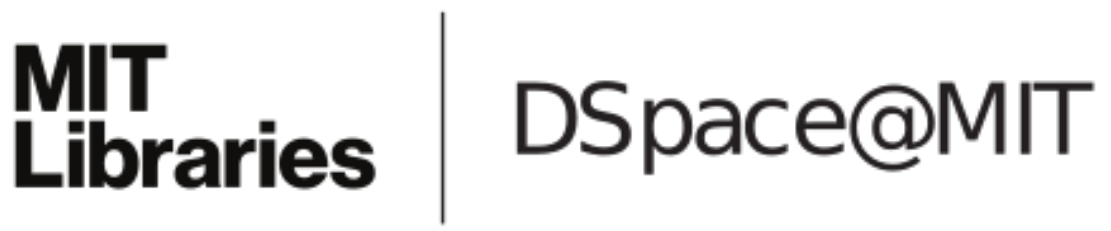

\author{
MIT Open Access Articles \\ Programmable and printable Bacillus subtilis \\ biofilms as engineered living materials
}

The MIT Faculty has made this article openly available. Please share how this access benefits you. Your story matters.

Citation: Huang, Jiaofang, Suying Liu, Chen Zhang et al. "Programmable and printable Bacillus subtilis biofilms as engineered living materials." Nature Chemical Biology, 15 (2019):34-41. () 2018, The Author(s).

As Published: https://dx.doi.org/10.1038/S41589-018-0169-2

Publisher: Springer Nature

Persistent URL: https://hdl.handle.net/1721.1/125850

Version: Author's final manuscript: final author's manuscript post peer review, without publisher's formatting or copy editing

Terms of use: Creative Commons Attribution-Noncommercial-Share Alike 


\title{
Programmable and printable Bacillus subtilis biofilms as engineered living materials
}

\author{
Jiaofang Huang ${ }^{1,9}$, Suying Liu ${ }^{1,2,9}$, Chen Zhang ${ }^{13,3,9}$, Xinyu Wang1, Jiahua Pu', Fang Ba4 , Shuai Xue ${ }^{5}$, \\ Haifeng Ye ${ }^{5}$, Tianxin Zhao', Ke Li', Yanyi Wang', Jicong Zhang', Lihua Wang 2,6, Chunhai Fan (1) 2,7, \\ Timothy K. Lu ${ }^{8}$ and Chao Zhong ${ }^{1 *}$
}

\begin{abstract}
Bacterial biofilms can be programmed to produce living materials with self-healing and evolvable functionalities. However, the wider use of artificial biofilms has been hindered by limitations on processability and functional protein secretion capacity. We describe a highly flexible and tunable living functional materials platform based on the TasA amyloid machinery of the bacterium Bacillus subtilis. We demonstrate that genetically programmable TasA fusion proteins harboring diverse functional proteins or domains can be secreted and can assemble into diverse extracellular nano-architectures with tunable physicochemical properties. Our engineered biofilms have the viscoelastic behaviors of hydrogels and can be precisely fabricated into microstructures having a diversity of three-dimensional (3D) shapes using 3D printing and microencapsulation techniques. Notably, these long-lasting and environmentally responsive fabricated living materials remain alive, self-regenerative, and functional. This new tunable platform offers previously unattainable properties for a variety of living functional materials having potential applications in biomaterials, biotechnology, and biomedicine.
\end{abstract}

B acterial biofilms, skeletal tissue, and other natural biological systems are multifunctional and environmentally responsive assemblages of living and nonliving components ${ }^{1}$. As these natural systems grow, self-repair, and adapt to the environment, they have distinctive 'living' attributes that are beyond the reach of the vast majority of existing synthetic materials ${ }^{2}$. Scientists and engineers have long envisioned harnessing these attributes to create dynamic, environmentally responsive, and tunable 'living functional materials' that incorporate both nonliving components and living cells ${ }^{3,4}$.

Biofilms are formed by bacteria on various surfaces via the synthesis and secretion of a cohesive and protective extracellular matrix that helps them tolerate harsh or nutrient-poor environments ${ }^{5}$. Scaffolds for bacterial biofilm formation often consist of amyloid fibers, proteins that self-assemble to form various cross-beta nanoarchitectures ${ }^{6,7}$. These amyloid fibers are known to function in a number of physiological processes required for bacterial growth and survival, including adhesion to diverse interfaces and/or host tissues $^{8}$, detoxification of toxic compounds ${ }^{9}$, resistance to antibiotics $^{10}$, morphological differentiation of filamentous bacteria ${ }^{11}$, and electron transport ${ }^{12}$. Recent bioengineering efforts have exploited amyloid fibers to develop both engineered cellular consortia and artificial biofilms ${ }^{1,11,13,14}$. For example, recent work has demonstrated the controllable and autonomous patterning of curli fibrils assembled in Escherichia coli cells ${ }^{1,15}$ and a biofilm-integrated nanofiber display (BIND) system; with this system the E. coli biofilm extracellular matrix can be programmed via manipulation of genetically engineered functional peptide domains of the CsgA protein ${ }^{14}$.
Despite these important advances, multiple technical challenges must be overcome before amyloid fibers can be used routinely and reliably in the design of living functional materials. In engineered biofilms research, known problems include the inability of bacterial export machinery to secrete large proteins (for example, the E. coli curli system is limited to secretion of short peptides or protein domains containing up to 59 amino acids ${ }^{14}$, which thereby reduces the scope of possible material functionalities, as well as difficulties with the controlled processing of such complex materials into customizable 3D structures with well-defined designed geometries ${ }^{16}$. Furthermore, considering that these materials are living, and given the potential risks that live bacteria present to human beings and the environment ${ }^{17}$, it is now appreciated that in addition to using bacterial species that are considered safe, the careful packaging of such materials into confined environments is a particularly important and necessary element of their design. Thus, perhaps it is unsurprising that the biomanufacture of programmable engineered biofilms remains a sparsely explored area of research.

Here, we used a combination of genetic engineering and biomanufacturing approaches to manipulate biofilms produced by Bacillus subtilis, a Gram-positive aerobic endospore-forming bacterium that is "generally regarded as safe" (GRAS) ${ }^{18}$. We developed a highly flexible and tunable living functional materials platform with several practical applications (Fig. 1). Specifically, we harnessed the TasA amyloid machinery of B. subtilis and developed genetically programmable TasA fusion proteins with diverse domain combinations offering versatile functionality for cell biology, enzymology, and other applications. These fusion proteins can be secreted and

'Materials and Physical Biology Division School of Physical Science and Technology, ShanghaiTech University, Shanghai, China. ${ }^{2}$ Division of Physical Biology and Bioimaging Center Shanghai Synchrotron Radiation Facility CAS Key Laboratory of InterfacialPhysics and Technology, Shanghai Institute of Applied Physics, Chinese Academy of Sciences, Shanghai, China. ${ }^{3}$ Shanghai Institute of Organic Chemistry, Chinese Academy of Sciences, Shanghai, China. ${ }^{4}$ School of Life Science and Technology, ShanghaiTech University, Shanghai, China. ${ }^{5}$ Shanghai Key Laboratory of Regulatory Biology, Institute of Biomedical Sciences and School of Life Sciences, East China Normal University, Shanghai, China. ${ }^{6}$ Shanghai Key Laboratory of Green Chemistry and Chemical Processes School of Chemistry and Molecular Engineering, EastChina Normal University, Shanghai, China. ${ }^{7}$ School of Chemistry and Chemical Engineering, and Institute of Molecular Medicine, Renji Hospital, School of Medicine, Shanghai Jiao Tong University, Shanghai, China. ${ }^{8}$ Synthetic Biology Group, Research Laboratory of Electronics, Massachusetts Institute of Technology, Cambridge, MA, USA. ${ }^{9}$ These authors contributed equally: Jiaofang Huang, Suying Liu, Chen Zhang. *e-mail: zhongchao@shanghaitech.edu.cn 
a

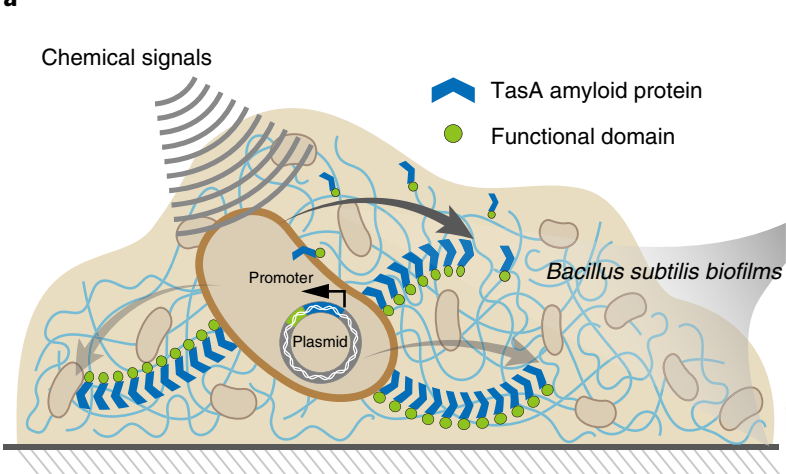

Programmable living functional materials b

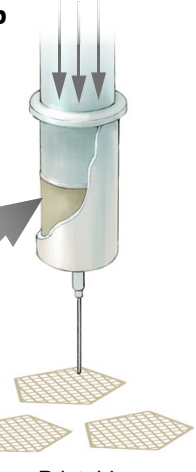

Printable

living materials
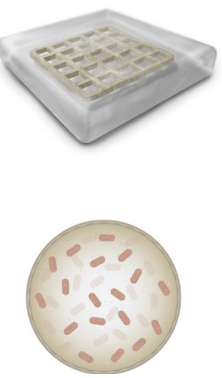

Encapsulated living materials

Fig. 1 | Design for a programmable and printable B. subtilis biofilm production platform. a, Schematic of the programmable living biofilm platform based on the Tas $A$ amyloid export machinery of $B$. subtilis. In the presence of chemical inducers, B. subtilis cells express rationally designed Tas $A$ fusion proteins comprising the amyloidogenic TasA domain (blue) and a tunable functional domain (green). Upon secretion, the fusion proteins self-assemble into extracellular fibrous networks that are closely associated with cell surfaces, resulting in programmable biofilms with tunable non-natural functional properties. b. Schematic of a printable living biofilm using a 3D printing technique. The living B. subtilis biofilms exhibit viscoelastic properties that make them suitable for 3D printing. c, Schematic showing that the living B. subtilis biofilms maintain their natural viability and various cellular capacities such as self-regeneration when trapped in hydrogels or microcapsules.

can self-assemble around living cells into diverse extracellular nanoarchitectures with tunable physiochemical properties (for example, viscoelasticity; Fig. 1a), as well as be flexibly configured in space (for example, 3D printing of living biofilms; Fig. 1b). Importantly, while retaining their natural viability and various cellular capacities like self-regeneration, these constructed living materials can also be designed to exhibit non-natural functionalities that are dependent on the incorporated functional domains, including altered enzymatic activity, red fluorescence, and the capacity for templated assembly of inorganic nanoparticles (NPs).

In a series of increasingly complex proof-of-concept demonstrations, we deployed these engineered biofilms in fluorescence detection, conjugation chemistry, single-substrate bioremediation, and multireaction bioremediation cascades incorporating NPs. We also exploited the intrinsic viscoelastic properties of our engineered biofilms and fabricated well-defined 'living shapes', trapping these materials into hydrogels and microgels using $3 \mathrm{D}$ printing and microencapsulation techniques (Fig. 1c). Finally, as these bacterial biofilms are composed of living cells in well-defined geometries, we evaluated their self-regeneration capacity, storage, and long-term viability. Our study demonstrates that we have constructed a programmable and printable living functional material platform that may enable new applications in nanomanufacturing, biocatalysis, biomedicine, and other technical fields.

\section{Results}

Design for a programmable $B$. subtilis biofilm platform. Unlike E. coli or other Gram-negative bacteria, B. subtilis has only one outer membrane, a feature that has long made this bacterium popular for the production of secreted enzymes and other proteins at an industrial scale ${ }^{18}$. Unlike the adherent $E$. coli cells that form biofilms at various interfaces, $B$. subtilis biofilms tend to form at air-water interfaces $^{6,19}$ and exhibit viscoelastic properties ${ }^{20}$ that are attractive for fabrication applications. The production of amyloid fibers in $B$. subtilis biofilms is tightly regulated by the $\operatorname{tap} A$-sip $W$-tas $A$ gene operon, which encodes TasA and TapA as major and minor amyloid components of biofilms, respectively. TapA protein acts as a molecular nucleator for the extracellular assembly of TasA protein into nanofibers on the cell surface, providing structural integrity to B. subtilis biofilms ${ }^{21}$. Notably, TasA protein differs from other biofilm-forming amyloid proteins by the apparent lack of repeats in its sequence ${ }^{22}$.
We focused our engineering efforts on TasA, because this amyloid family protein is well known for having high tolerance to chemical elaboration and functionalization without disruption of its self-assembly capacity ${ }^{23}$. To assess whether genetically modified TasA can be secreted and can self-assemble into extracellular nanofibers, we first established a platform for the inducible transcriptional and translational control over the expression of variously functionalized genetically engineered TasA variants (Fig. 2a and Supplementary Table 2). We used IPTG-inducible expression plasmids to express the engineered variants ("TasA-R") and used these plasmids to transform a $B$. subtilis strain that we had constructed (2569 $\Delta \operatorname{tas} A \Delta \sin R \Delta e p s)$. We designed this strain to facilitate the detection of functionalized TasA-R variants, as well as to lack (1) the $\operatorname{sinR}$ gene, which is known to limit biofilm production by repressing the eps and tas $A$ operons, and (2) the epsA-O gene cluster, which encodes exopolysaccharides (EPS).

Consistent with our design, the biofilm-defective strain $B$. subtilis $2569 \Delta \operatorname{tas} A \Delta \sin R \Delta e p s(\Delta \operatorname{tas} A \Delta \sin R \Delta e p s)$ did not produce extracellular TasA fibers (Fig. 2b), but upon IPTG induction the $\Delta \operatorname{tas} A \Delta \sin R \Delta$ eps cells harboring the exogenous expression plasmid pHT01 for the TasA-R variants (for example, TasA-mCherry) restored amyloid fiber production. Amyloid production was confirmed by transmission electron microscopy (TEM), which showed that immuno-gold-labeled anti-TasA antibodies could specifically bind to the TasA-R fibrous networks (Fig. $2 \mathrm{c}-\mathrm{m}$ and Supplementary Fig. 1). Biofilm production was further confirmed using a standard crystal-violet (CV) assay, in which the presence of IPTG increased the biomass for each of the TasA-R variants compared to their controls without IPTG induction (Supplementary Fig. 2). In addition, we also compared the morphologies and diameters of the differently functionalized nanofibers (TasA-HisTag, TasA-Mefp5, and TasA-OPH as representatives) based on TEM imaging and found that there was an obvious trend in the diameters of the different types of nanofibers: the diameter of the nanofibers increased as the size of the monomer fusion domains increased (TasA-OPH $(18.7 \pm 2.3 \mathrm{~nm})>$ TasA-Mefp5 $\quad(15.7 \pm 2.0 \mathrm{~nm})>$ TasA-HisTag $(13.6 \pm 1.6 \mathrm{~nm})$ ) (Supplementary Fig. 3).

Functional performance of the B. subtilis biofilms. We next assessed whether the biofilms containing the secreted TasA-R nanofibers had new functional properties as a result of their various engineered 

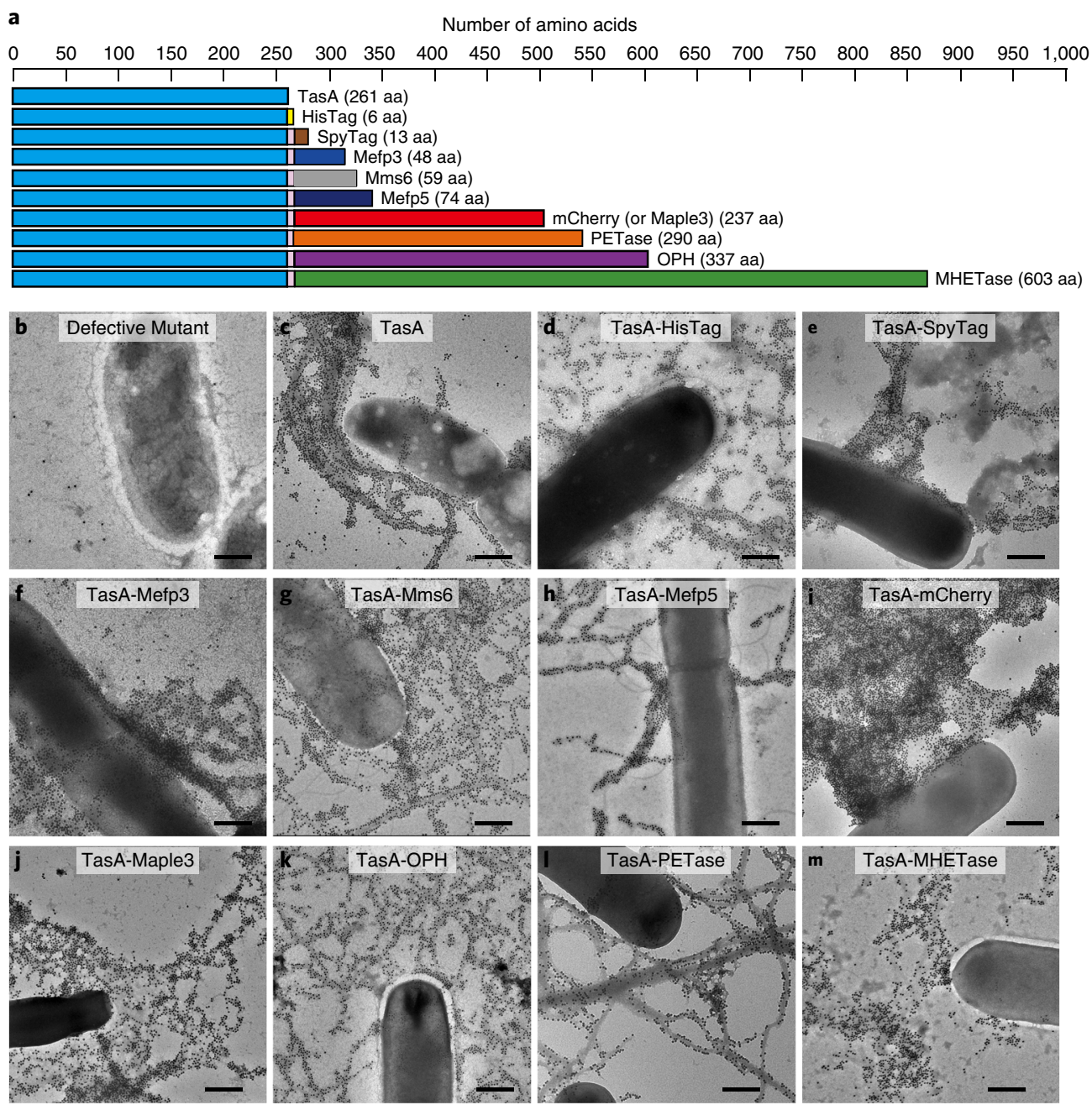

Fig. 2 | Extracellular secretion and assembly of TasA-R amyloid proteins into fibrous networks associated with the surfaces of engineered B. subtilis cells. a, A series of TasA-R fusion constructs comprising TasA backbone (261 amino acids (aa)) and functional R domain (with variable number of amino acids) were rationally designed and applied in this study: $\operatorname{HisTag}^{37}$ ( 6 or $7 \mathrm{aa}$ ); $\operatorname{SpyTag}^{24}$ (13 aa), a short peptide derived from Streptococcus pyogenes that specifically reacts with its protein partner SpyCatcher; Mefp3 (48 aa) and Mefp5 (74 aa), adhesive mussel foot proteins derived from Mytilus edulis ${ }^{38}$; Mms6 (59 aa), a peptide for magnetite templating ${ }^{39}$; mCherry ${ }^{40}$ and Maple3 (ref. ${ }^{41}$, (237 aa), fluorescent proteins; PETase (290 aa), a poly (ethylene terephthalate) (PET) hydrolase; OPH, organophosphate hydrolase ${ }^{42}$ (337 aa); and MHETase (603 aa), mono (2-hydroxyethyl) terephthalic acid (MHET) hydrolase ${ }^{25} \cdot \mathbf{b}-\mathbf{m}$, Morphologies of the assembled TasA-R fibers associated with cell surfaces based on immunogold labeling and transmission electron microscopy (TEM): control strain (b); TasA (c); TasA-HisTag (d); TasA-SpyTag (e); TasA-Mefp3 (f);TasA-Mms6 (g); TasA-Mefp5 (h); TasA-mCherry (i); TasA-Maple3 (j); TasA-OPH (k); TasA-PETase (I) and TasA-MHETase ( $\mathbf{m})$. The TEM samples were collected from the $\Delta \operatorname{tas} A \Delta \operatorname{sinR} \Delta$ eps strain harboring a plasmid that expresses fusion proteins of interest under the control of an IPTG-inducible promoter. A gold-decorated anti-TasA antibody was used for labeling. Scale bars, $500 \mathrm{~nm}$. TEM imaging was repeated three times for each sample with similar results obtained based on three independently cultured biofilm samples of the same type $(n=3)$.

fusion domains. We started with the TasA-R variants fused to the mCherry protein (TasA-mCherry) and found that, upon IPTG induction, biofilms expressing TasA-mCherry nanofibers exhibited red fluorescence, validating the concept that mCherry retains its function when expressed in a biofilm generated by our platform (Fig. 3a and Supplementary Fig. 4). We next generated TasA-R variants that use the covalent isopeptide bond formation reaction between the SpyTag (TasA-SpyTag) and SpyCatcher protein binding partners ${ }^{24}$ as a fusion with the fluorescent protein GFP as a readout (SpyCatcher-GFP). We observed green fluorescence only when we added free SpyCatcherGFP protein to biofilms containing TasA-SpyTag nanofibers (but not with TasA-HisTag nanofibers or other controls), demonstrating that the observed fluorescence in TasA-SpyTag nanofibers was samplespecific, and the fused SpyTag indeed remained functional in the form of TasA-SpyTag nanofibers (Fig. 3b and Supplementary Fig. 5).
To further explore the capacities of our programmable biofilm platform, we engineered a strain producing extracellular TasAMHETase nanofibers. MHETase-an enzyme that degrades the toxic industrial compound MHET (mono (2-hydroxyethyl) terephthalic acid) into the less toxic TPA (terephthalic acid)-was purposely chosen here as a test not only for its practical use but also because it is quite large (603 amino acids) ${ }^{25}$. Upon IPTG induction, the cells produced and assembled TasA-MHETase nanofibers on their surfaces (Fig. 2m). We next treated these IPTG-induced biofilms with free MHET and used HPLC-MS to detect TPA. Our results confirmed that the TasA-MHETase nanofibers retained their MHETase enzymatic activity (Fig. 3c and Supplementary Fig. 6). The relationship between catalytic rate and substrate concentration in the TasA-MHETase biofilm system followed typical MichaelisMenten kinetics (Supplementary Fig. 6c). Notably, although 
a

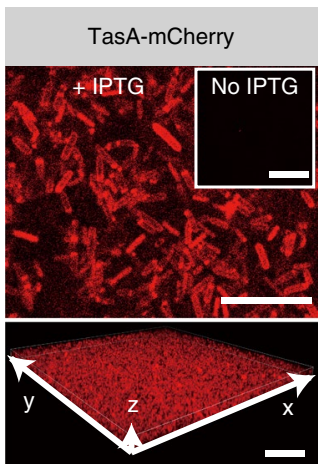

b

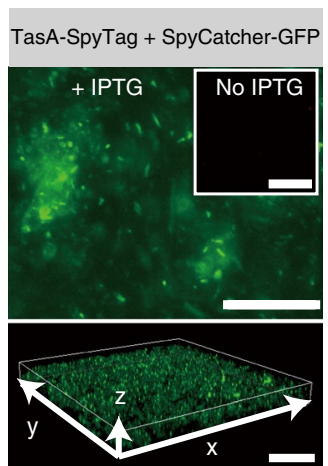

d

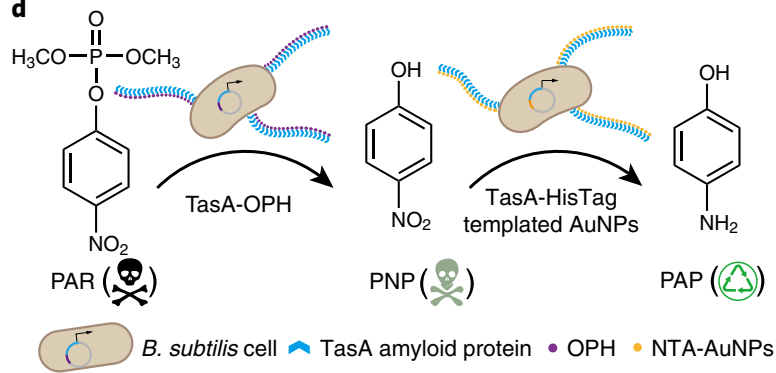

e

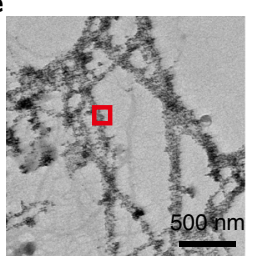

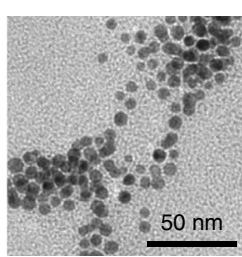

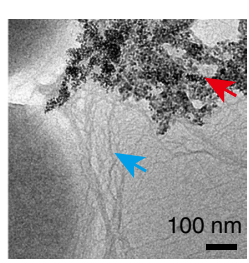

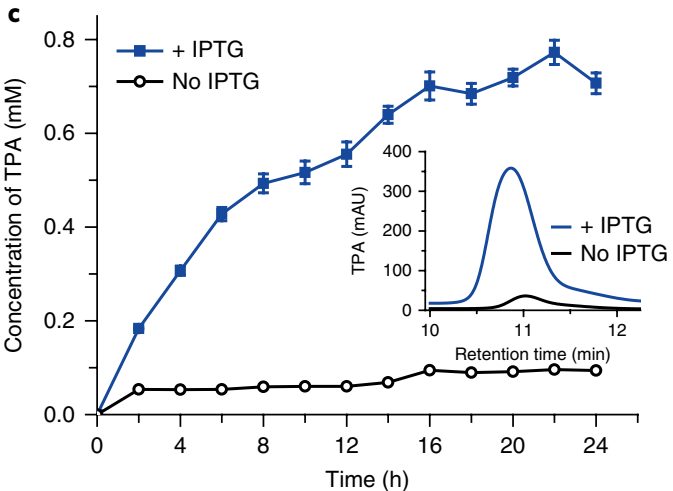
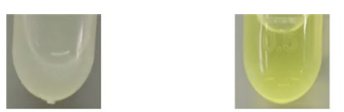

$(2 \mathrm{~h})$

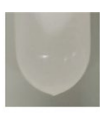

$(4 \mathrm{~h})$

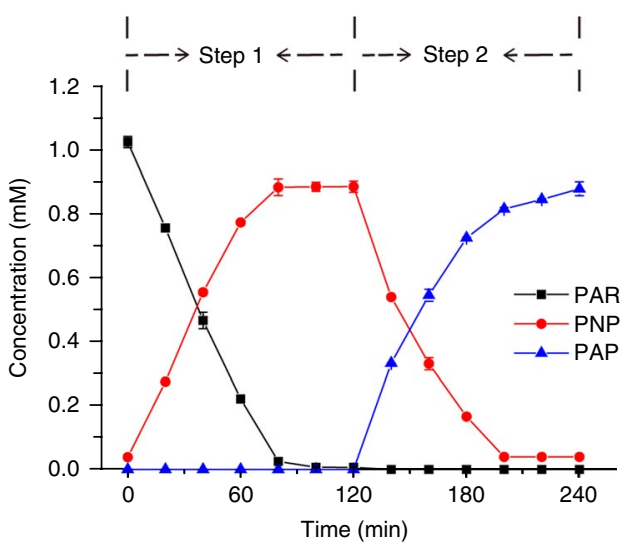

Fig. 3 | Functional characterization of engineered B. subtilis biofilms. a, Confocal microscopic image of a $\Delta \operatorname{tas} A \Delta \operatorname{sinR} \Delta$ eps/Tas A-mCherry biofilm. b. Confocal microscopic image showing green fluorescence emitted from a TasA-SpyTag nanofiber biofilm to which SpyCatcher-GFP protein binding partners were covalently attached via isopeptide bonds. Scale bars, $10 \mu \mathrm{m}$ in $\mathbf{a}$ and b. c. HPLC assay for MHET degradation with a $\Delta$ tasA $\Delta$ sinR $\Delta$ eps/TasAMHETase biofilm. Results show means \pm s.e.m. of three independent biofilm cultures $(n=3)$. d, Biodegradation of organophosphate pesticides through a two-step biocatalytic cascade reaction using co-cultured strains for a TasA-OPH biofilm and a TasA-HisTag biofilm. The functional OPH enzyme present in the TasA-OPH biofilm degrades PAR into PNP. Next, AuNPs that have been immobilized on a TasA-HisTag biofilm further degrade PNP to PAP. $\mathbf{e}$, TEM images showing that Ni-NTA-decorated AuNPs were anchored onto biofilms containing TasA-HisTag nanofibers (left and middle) and complex biofilms containing TasA-OPH (blue arrow) and TasA-HisTag nanofibers, anchored with AuNPs (red arrow; right). The middle image is a zoomed-in area (marked with a red square) of the left image. $\mathbf{f}$, Concentrations of PAR, PNP, and PAP in solution over time. The inserted pictures represent samples collected at different stages. All results in $\mathbf{a}, \mathbf{b}, \mathbf{e}$ and $\mathbf{f}$ were repeated three times for each sample with similar results based on three independent biofilm cultures.

engineered E. coli biofilms with single enzymatic capacities have been reported, such examples required the use of covalent reactions between displayed SpyTags on biofilms and separately purified SpyCatcher-enzyme fusions ${ }^{26}$, a multistep process that involves considerably more labor than our single-step IPTG induction of B. subtilis TasA-MHETase biofilms.

Having demonstrated a single enzyme application, we next tested whether our programmable biofilm platform could be used for multistep reactions (i.e., biocatalytic cascades) to degrade organophosphate pesticides into harmless chemicals. We chose a two-step cascade consisting of (i) the organophosphate hydrolase (OPH)catalyzed degradation of the pesticide paraoxon (PAR) into the less harmful intermediate product paranitrophenol (PNP), and (ii) a reaction catalyzed by HisTag-immobilized gold NPs (AuNPs) in which PNP is further degraded into harmless $\mathrm{p}$-aminophenol (PAP) (Fig. 3d). The TasA-HisTag nanofibers use His-metal-coordination chemistry to promote the assembly of AuNPs (Fig. 3e).

We therefore engineered two separate strains that respectively expressed and assembled functional TasA-OPH and TasA-HisTag nanofibers (Fig. 2d,k). We first confirmed that a biofilm containing only TasA-OPH nanofibers could efficiently catalyze the PAR-toPNP reaction and that a biofilm containing only the TasA-HisTag nanofibers in the presence of AuNPs and $\mathrm{NaBH}_{4}$ could efficiently catalyze the PNP-to-PAP reaction. Though this PNP degradation generally followed first-order reaction kinetics, it was interesting to note that the TasA-HisTag-immobilized NPs actually outperformed free NPs (control reaction in aqueous solution) for the degradation of PNP (Supplementary Fig. 7). This surprising finding can perhaps be ascribed to enhanced absorption of PAR onto the biofilm. We next co-cultured the strains to produce a single, hybrid biofilm that contained both the TasA-OPH nanofibers (enzyme-mediated degradation) and the TasA-HisTag nanofibers (NP-mediated degradation). With both AuNPs and $\mathrm{NaBH}_{4}$ present, each of the two degradation reactions of the cascade exhibited kinetics similar to those of the separate reactions for the single-strain biofilms (Fig. 3f).

The complex and heterogeneous features of the fibrous networks in the biofilm of this co-cultured hybrid reaction system were assessed with TEM imaging, confirming that AuNPs specifically bound to TasA-HisTag nanofibers rather than to TasA-OPH nanofibers (Fig. 3e). The immobilized AuNPs on the nanofibers circumvent 
direct contact of NPs with bacteria, preventing potential damage to the cells caused by free $\mathrm{NPs}^{27}$. Thus, our programmable B. subtilis biofilm platform can be used to make hybrid catalytic systems coupling living biofilms with inorganic NPs. The ability to grow biofilms comprising multiple strains and to use hybrid reaction systems in designs that merge the various advantages of each component highlights the promise of developing yet more complex and multicomponent biofilms as eco-friendly, high-efficiency, and self-regenerating catalytic systems for a potentially wide range of applications.

Viscoelastic properties and printability. Having demonstrated that our platform can be used to make multistrain biofilms capable of complex multistep chemistries, we next explored whether we could finely manipulate biofilm geometries. The ability to precisely control the physical context for complex chemical reactions would have obvious utility in nanomanufacturing and materials applications $\mathbf{s}^{16,28,29}$. Wild-type $B$. subtilis biofilms are known to form complex gel-like materials at water-air interfaces and on solid agar plates with viscoelastic properties, which in theory would be attractive for many biofabrication applications like printing. We therefore characterized the viscoelastic properties of wild-type biofilms and tested whether we could alter the viscoelastic properties of engineered biofilms for biofabrication. We first examined the surface morphology of wild-type and TasA-HisTag biofilms and noted that the engineered biofilm was smoother than the typical wrinkled surface of the wild-type biofilm (Fig. 4a,b). Analysis with a goniometer revealed that the engineered biofilm was much more hydrophilic than the wild-type biofilm (water contact angle of $25.1 \pm 5.0^{\circ}$ for TasA-HisTag biofilms versus $124.2 \pm 3.0^{\circ}$ for the wild-type biofilm) (Fig. 4a,b). The engineered biofilm was based on a $\Delta \operatorname{tas} A \Delta \sin R \Delta e p s$ mutant strain (by removing the epsA-O gene cluster and tas $A$ gene from the wild-type strain); as anticipated, the lack of the endogenous biofilm component pathways dramatically reduced the hydro-

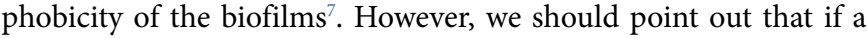
future application requires more hydrophobicity, there are at least two approaches that can restore the hydrophobic properties of the biofilms: (i) adding those endogenous systems back into the host strain, and/or (ii) functionalizing fusion domains onto TasA with hydrophobic features.

We next turned to fabricating biofilms into defined geometries using $3 \mathrm{D}$ printing techniques. When we printed regular pentagon shapes using the wild-type and TasA-HisTag biofilms with the same printing conditions (nozzle diameter set to $160 \mu \mathrm{m}$ ), the lines of the wild-type biofilm expanded to $500 \mu \mathrm{m}$ as they were deposited, whereas the TasA-HisTag biofilm had the desired width $(160 \mu \mathrm{m})$ (Fig. 4c,d). Similar trends were found with other engineered biofilms, such as TasA-mefp5 and TasA-OPH biofilms, which all exhibited better printability than the wild-type biofilm (Supplementary Fig. 8). Detailed rheological measurements and comparisons of the storage modulus ( $\mathrm{G}^{\prime}$, representing elastic deformation) and loss modulus ( $\mathrm{G}^{\prime \prime}$, representing viscous deformation) as well as the capacity of instantaneous recovery of the viscoelastic networks ${ }^{30}$ suggested that the relatively low viscoelasticity of the engineered biofilms made them more suitable for printing than wild-type biofilm (Fig. 4e,f and Supplementary Figs. 9 and 10). Specifically, the internal network structures of the two hydrogel-like biofilms were subjected to similar compression when they were exposed to the extremely high pressures in the printer's cavity before being extruded; however, the differential viscoelastic properties of the two biofilms would respond to this pressure discretely: given the higher storage modulus and stronger instantaneous recovery capacity of its viscoelastic networks, the wild-type biofilm could be expected to possess better reversible deformability (shape recovery) than the engineered TasA biofilm, and thus would exhibit 'expanded' line features as it was extruded. In addition, we speculate that the richness of EPS polysaccharide components in the wild-type biofilm may also contribute to the observed 'expanded' line feature, as they may promote moisture absorption. The wild-type biofilm might have the EPS components fully exposed to air, as their hydrophobic protein layer could be disrupted during biofilm scraping and printing processes. Collectively, our results demonstrated that the viscoelastic properties of $B$. subtilis biofilms could be tunably altered, and thus the engineered biofilms could be manipulated at high resolution for 3D printing applications (Fig. 4d and Supplementary Fig. 8).

We next explored the controllable geometry and functionality of our engineered B. subtilis biofilms by using several types of inorganic NPs. Specifically, B. subtilis biofilms containing TasA-HisTag nanofibers with diverse immobilized quantum dots (QDs) were used as building materials to fabricate well-defined shapes with 3D printing techniques. We prepared building materials in three colors by adding blue (CdZnS@ZnS), red (CdSeS@ZnS), and green(CdZnSeS@ ZnS) QDs to TasA-HisTag nanofiber biofilms. When printed into different 3D structures, such as polygons and letters, these cell/inorganic hybrid materials were blue, red, and green under UV light $(350 \mathrm{~nm})$, respectively (Fig. $4 \mathrm{~g}$ and Supplementary Fig. 11). The fluorescence of these materials was confirmed by confocal fluorescence microscopy and fluorescence spectrometry (Fig. 4h,i).

Self-regeneration, encapsulation, and viability. One of the most attractive attributes of living materials is their ability to self-regenerate. To assess whether our engineered biofilms maintain this ability, we printed TasA-HisTag nanofiber biofilms into controlled hexagonal patterns on glass slides and then placed the printed biofilms in direct contact with a nutrient-rich agar plate (Fig. 5a). Encouragingly, the biofilms regrew in hexagonal patterns after $2 \mathrm{~d}$ on agar plates. We then exposed the biofilm on the first agar plate to a second agar plate and found that the biofilm still grew in the initial hexagonal pattern, demonstrating the reasonably high-fidelity, self-regenerative capacity of our engineered and printed biofilms (Fig. 5b). Remarkably, analysis of the viscoelastic properties of the regenerated biofilms showed that they had almost the same elastic and viscous modulus levels as the initially grown biofilms (Fig. $5 \mathrm{c}$ and Supplementary Fig. 12), a finding emphasizing that not only the genotype of a given biofilm but also its phenotypic features (including material properties) can be regenerated.

We next assessed the viability of hybrid functionalized biofilms. Preliminarily, we cultured control (biofilm-deficient), TasA, or TasAHisTag biofilms in the presence of equal amounts of QDs and then monitored biofilm production (Supplementary Fig. 13). We found that the presence of QDs did not deleteriously affect the growth and production of TasA-HisTag biofilms; however, the QDs did inhibit the growth of both biofilm-deficient bacteria and biofilms lacking the HisTag modification. Moreover, TEM imaging further revealed that toxic QDs could be immobilized onto TasA-HisTag nanofibers, avoiding direct contact with bacterial cells (Supplementary Fig. 14). These results implied that the HisTag could interact with and immobilize the QDs onto biofilms and thereby protect the functionalized biofilm from direct damage by these toxic nanoparticles. Indeed, a self-regeneration experiment testing regrowth on agar plates showed that the cells of hybrid functionalized biofilms remained viable after exposure to QDs (Supplementary Fig. 15). We also confirmed that our biofilms remained viable following various fabrication techniques, including printing into various shapes on glass and polyvinyl chloride (PVC) polymers, among others (Supplementary Fig. 11).

To further demonstrate the potential of our engineered biofilms for biofabrication, we encapsulated the biofilms into geometrically predefined hydrogel compartments fabricated by two techniques: $3 \mathrm{D}$ printing and microencapsulation. We then evaluated both their functionality and their viability in these confined environments by sequentially printing hydrogels and a strain that can produce a TasA-mCherry nanofiber biofilm. Specifically, the printed biofilm was sandwiched between two layers of hydrogels consisting of 


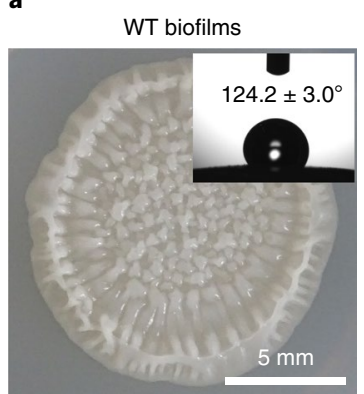

C

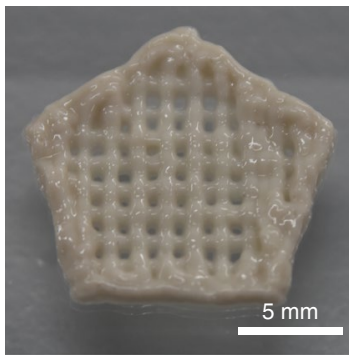

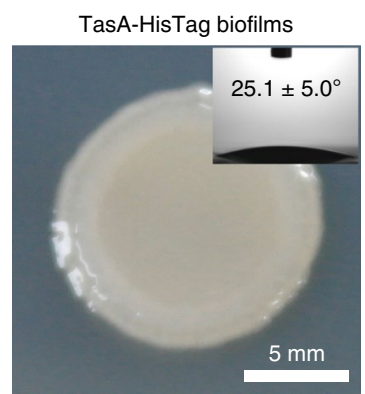

d

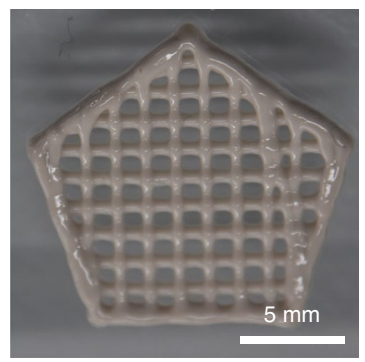

e

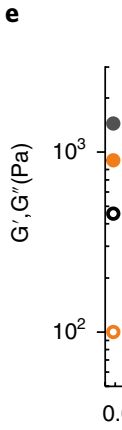

$\mathbf{f}$

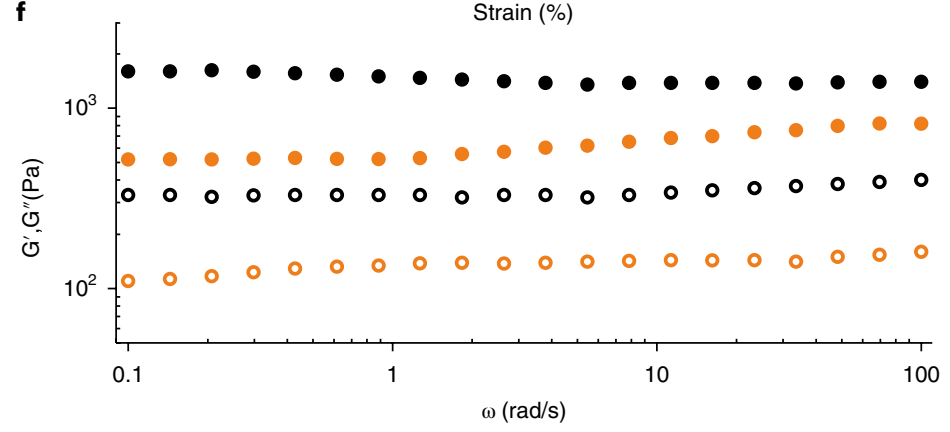

h

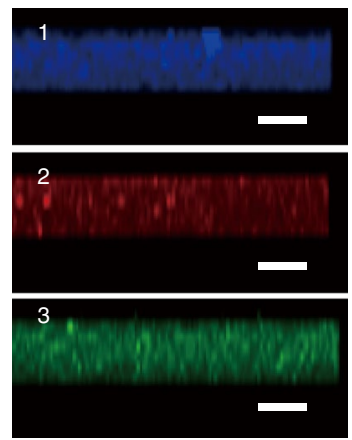

G" of WT biofilms

$\mathrm{G}^{\prime \prime}$ of TasA-HisTag biofilms
$\mathrm{G}^{\prime}$ of TasA-HisTag biofilms o

000000000000000000000

$\circ \circ \circ \circ \circ \circ \circ \circ \circ \circ 000000^{0} 0^{8}$

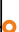

,

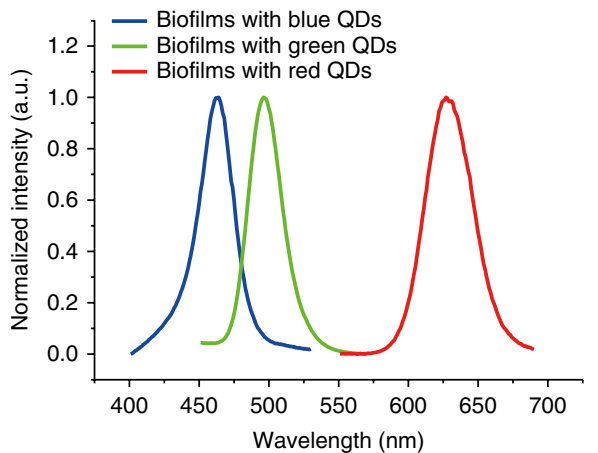

Fig. 4 | Morphology, rheological properties, and 3D printing of engineered B. subtilis biofilms. a,b, Digital camera image showing the general morphology of the wild-type biofilm (a) and the TasA-HisTag biofilm (b); water contact assay is also shown (insets). c, 3D-printed wild-type biofilm. d, 3D-printed TasA-HisTag biofilm. e,f, Comparison of rheological properties of the wild-type biofilm and the TasA-HisTag biofilm, measured by strain sweep experiments (e; strain, 0.01 to 100\%; frequency, 1 rad/s) and frequency sweep experiments (f; frequency, 0.1 to $100 \mathrm{rad} / \mathrm{s}$; strain, 1\%). g, 3D complex printing of TasA-HisTag biofilms and inorganic NPs into diverse patterns. Shown are digital photographs of the printed complex structures taken under normal (top) and UV light (bottom). h,i, Fluorescence images (h) and fluorescence spectra (i) of the corresponding complex structures shown in $\mathbf{g}$. Note: the numbers 1, 2, and 3 specifically designated in $\mathbf{g}$ and $\mathbf{h}$ refer to the TasA-HisTag biofilms with the following types of immobilized quantum dots (QDs): (1) blueCdZnS@ZnS QDs, (2) greenCdZnSeS@ZnS QDs, and (3) red Co-NTA CdSeS@ZnS QDs, with maximum emission peak wavelengths at 464, 496, and $627 \mathrm{~nm}$, respectively. Scale bars, $5 \mathrm{~mm}$ in $\mathbf{g}$ and $5 \mu \mathrm{m}$ in $\mathbf{h}$. Results in $\mathbf{a}-\mathbf{d}$ and $\mathbf{g}$-i were repeated twice independently with similar results. Water contact angles inserted in $\mathbf{a}$ and $\mathbf{b}$ show means \pm s.e.m.based on three independent biofilm cultures $(n=3)$. Experiments in $\mathbf{e}$ and $\mathbf{f}$ were repeated three times with similar results based on biologically independent biofilm cultures $(n=3)$.

gelatin and sodium alginate, two common types of polymeric materials used for hydrogel formation ${ }^{31,32}$. When immersed in minimal medium (MSgg) supplemented with $0.5 \mathrm{mM}$ IPTG, the biofilm trapped inside the gel exhibited red fluorescence (Fig. $5 \mathrm{~d}$ ). Remarkably, the biofilms encapsulated in the hydrogels remained viable and functional even after 5 weeks (without any supplemental nutrition), implying that the biofilms produced with our platform can exhibit long-term viability and do not require extensive energy inputs; these are very attractive attributes for the practical application of living functional materials (Supplementary Fig. 16).

We next looked into the fabrication of engineered biofilms using microencapsulation, a technique that had previously been used for encapsulation of mammalian cells inside alginate-poly-(L-lysine)alginate (APA) hydrogel microspheres ${ }^{33,34}$. Here, using a standard microencapsulation protocol, we were able to produce uniform APA/ $\mathrm{Ca}^{2+}$ hydrogel microspheres ( 400 microns) by mixing APA and cells (Fig. 5e). The encapsulated cells could sense IPTG molecules, producing biofilms that exhibited red fluorescence. This result demonstrated that the biofilm retained its functionality in the APA/Ca ${ }^{2+}$ microspheres (Fig. 5e and Supplementary Fig. 17). Thus, our programmable $B$. subtilis production platform can be used to produce functional and viable biofilms that are highly scalable, from simple growth in standard flasks and petri dishes to complex printed geometries and sophisticated microencapsulated environments.

\section{Discussion}

Recent studies have reported the incorporation of genetically engineered bacteria into 3D-printed hydrogels to create living wearable 


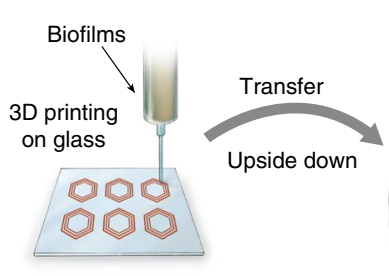

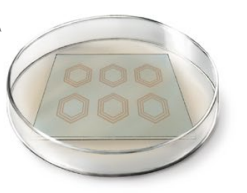

Self-regeneration on agar plate d

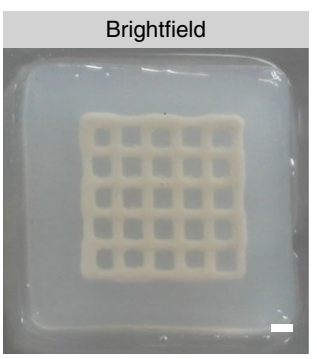

e

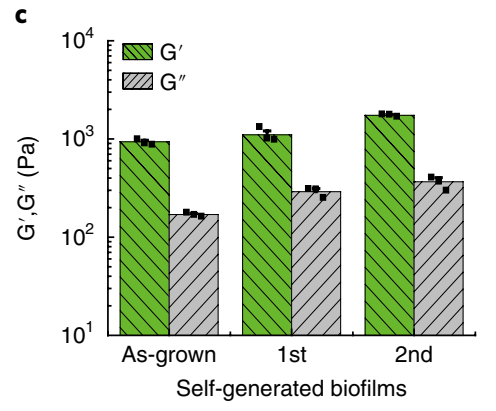

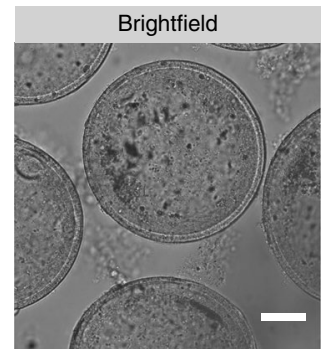

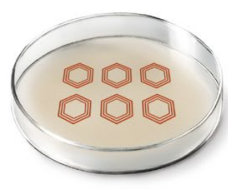

Self-generated biofilms
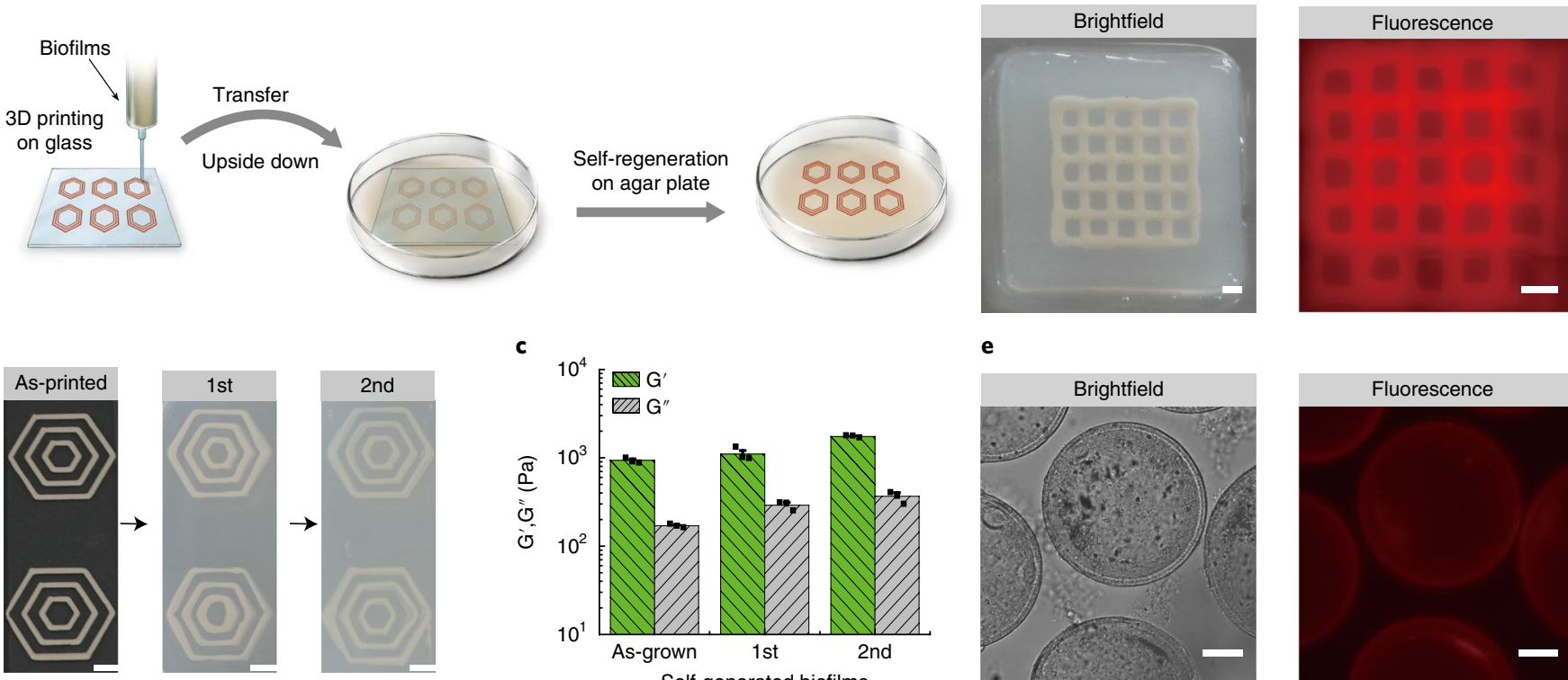

Fig. 5 | Self-regeneration, biofabrication, long-term viability, and functional performance of hydrogel-trapped B. subtilis biofilms. a, 3D printing of engineered $B$. subtilis biofilms into patterned structures on glass slides and self-regeneration of patterned biofilms on agar plates for two continuous generations: cartoon shows the 3D printing and self-regeneration processes. $\mathbf{b}$, For the self-regeneration experiments, the 3D printed samples were placed upside down on an agar plate. From left to right: the printed patterns on glass slide, the biofilms regrowing in the hexagonal patterns on the first nutrient-rich agar plate, and the biofilm still growing in the initial hexagonal patterns on the second nutrient-rich agar plate. c, Comparison of the storage modulus $\left(\mathrm{G}^{\prime}\right)$ and the loss modulus $\left(\mathrm{G}^{\prime \prime}\right)$ of the initially grown and self-regenerated biofilms. d,e, Functional performance of the biofabricated biofilms trapped inside a mixed hydrogel containing $5 \% \mathrm{w} / \mathrm{v}$ gelatin and $2 \% \mathrm{~W} / \mathrm{v}$ sodium alginate (d) or microencapsulated inside alginate-poly-( $\mathrm{L}-$-lysine)-alginate (APA) microcapsules (e). In both cases, when immersed into a solution containing IPTG, the trapped biofilms emitted red fluorescence throughout a 5-d culture period. The encapsulation of biofilms in alginate hydrogel microspheres was based on a microencapsulation technique ${ }^{34}$. Fluorescence images were acquired with the $587-\mathrm{nm}$ channel. Scale bars, $5 \mathrm{~mm}$ in $\mathbf{b} ; 1 \mathrm{~mm}$ in $\mathbf{d}$; and $100 \mu \mathrm{m}$ in $\mathbf{e}$. All data points in c were averages of three independent biofilm cultures with s.e.m shown $(n=3)$.

devices $^{2,17,20,28}$, but their fabrication requires the use of synthetic materials with appropriate viscoelastic properties, because the printability of such living materials devices depends primarily on the rheological properties of the synthetic materials. In this regard, printable bacterial biofilms have intrinsic advantages over free bacterial cells. First, the cells in biofilms are held in place by the extracellular network and are thus in some sense immobilized and partially protected against harsh conditions $\mathrm{s}^{35,36}$. Second, the gel-like biofilms can be engineered to possess tunable viscoelastic properties that are fit for printing in their own right. We envision that our programmable B. subtilis biofilm production platform could be suitable for use in medical implants, biosensors, and drug-delivery devices, as these applications require the secretion of viscous proteins or protein drugs, or the functional capacity to express other useful molecules.

Living functional materials represent an opportunity to harness engineered biological systems for new capabilities. By leveraging the power of genetic engineering and the intrinsic advantage of the export machinery of $B$. subtilis, we have introduced a new living functional material platform based on B. subtilis biofilms. We engineered the extracellular amyloid-like protein TasA, a major subunit of the fibrous components of B. subtilis biofilms, by fusing it with various other proteins or protein domains to endow the living system with new functionalities. We used our platform to make programmable living materials exhibiting functional properties as diverse as intrinsic fluorescence, enzymatic activity, and the templated assembly of various functional inorganic NPs to perform hybrid enzyme-inorganic reaction cascades. We also made living materials that exhibit attractive viscoelastic properties and fabricated them into diverse shapes and microstructures, both on their own and with other polymeric gel materials, using 3D printing and micro-gel encapsulation techniques. Our programmable and printable $B$. subtilis biofilms therefore represent a new type of living functional material: they are multifunctional, self-regenerating, and tunable, and have considerable fabrication processability. As this new type of living functional material offers previously unattainable material performance properties relevant to manufacturing, our study opens the door for the development of many conceivable new classes of complex multifunctional materials and dynamic and regenerative nanotechnologies.

\section{Online content}

Any methods, additional references, Nature Research reporting summaries, source data, statements of data availability and associated accession codes are available at https://doi.org/10.1038/ s41589-018-0169-2

Received: 8 May 2018; Accepted: 9 October 2018;

Published online: 03 December 2018

\section{References}

1. Chen, A. Y. et al. Synthesis and patterning of tunable multiscale materials with engineered cells. Nat. Mater. 13, 515-523 (2014).

2. Nguyen, P. Q., Courchesne, N. D., Duraj-Thatte, A., Praveschotinunt, P. \& Joshi, N. S. Engineered living materials: prospects and challenges for using biological systems to direct the assembly of smart materials. Adv. Mater. 30, e1704847 (2018)

3. Ball, P. Synthetic biology-engineering nature to make materials. MRS Bull. 43, 477-484 (2018).

4. Wang, Y., Pu, J., An, B., Lu, T. K. \& Zhong, C. Emerging paradigms for synthetic design of functional amyloids. J. Mol. Biol. 430, 3720-3734 (2018). 
5. Tallawi, M., Opitz, M. \& Lieleg, O. Modulation of the mechanical properties of bacterial biofilms in response to environmental challenges. Biomater. Sci. 5 887-900 (2017).

6. Vlamakis, H., Chai, Y., Beauregard, P., Losick, R. \& Kolter, R. Sticking together: building a biofilm the Bacillus subtilis way. Nat. Rev. Microbiol. 11 , 157-168 (2013).

7. Barnhart, M. M. \& Chapman, M. R. Curli biogenesis and function. Annu. Rev. Microbiol. 60, 131-147 (2006).

8. DeBenedictis, E. P., Liu, J. \& Keten, S. Adhesion mechanisms of curli subunit CsgA to abiotic surfaces. Sci. Adv. 2, e1600998 (2016).

9. Edwards, S. J. \& Kjellerup, B. V. Applications of biofilms in bioremediation and biotransformation of persistent organic pollutants, pharmaceuticals/ personal care products, and heavy metals. Appl. Microbiol. Biotechnol. 97, 9909-9921 (2013)

10. Donato, V. et al. Bacillus subtilis biofilm extends Caenorhabditis elegans longevity through downregulation of the insulin-like signalling pathway. Nat. Commun. 8, 14332 (2017).

11. Hobley, L., Harkins, C., MacPhee, C. E. \& Stanley-Wall, N. R. Giving structure to the biofilm matrix: an overview of individual strategies and emerging common themes. FEMS Microbiol. Rev. 39, 649-669 (2015).

12. Yates, M. D. et al. Measuring conductivity of living Geobacter sulfurreducens biofilms. Nat. Nanotechnol. 11, 910-913 (2016).

13. Cao, Y. et al. Programmable assembly of pressure sensors using patternforming bacteria. Nat. Biotechnol. 35, 1087-1093 (2017).

14. Nguyen, P. Q., Botyanszki, Z., Tay, P. K. \& Joshi, N. S. Programmable biofilm-based materials from engineered curli nanofibres. Nat. Commun. 5, 4945 (2014).

15. Wang, X. et al. Programming cells for dynamic assembly of inorganic nano-objects with spatiotemporal control. Adv. Mater. 30, 1705968 (2018).

16. Liu, X. et al. 3D printing of living responsive materials and devices. Adv. Mater. 30, 1704821 (2018).

17. Liu, X. et al. Stretchable living materials and devices with hydrogel-elastomer hybrids hosting programmed cells. Proc. Natl Acad. Sci. USA 114, 2200-2205 (2017).

18. Liu, L. et al. Developing Bacillus spp. as a cell factory for production of microbial enzymes and industrially important biochemicals in the context of systems and synthetic biology. Appl. Microbiol. Biotechnol. 97, 6113-6127 (2013)

19. Cairns, L. S., Hobley, L. \& Stanley-Wall, N. R. Biofilm formation by Bacillus subtilis: new insights into regulatory strategies and assembly mechanisms. Mol. Microbiol. 93, 587-598 (2014).

20. Kesel, S. et al. Direct comparison of physical properties of Bacillus subtilis NCIB 3610 and B-1 biofilms. Appl. Environ. Microbiol. 82, 2424-2432 (2016).

21. Driks, A. Tapping into the biofilm: insights into assembly and disassembly of a novel amyloid fibre in Bacillus subtilis. Mol. Microbiol. 80, 1133-1136 (2011).

22. Diehl, A. et al. Structural changes of TasA in biofilm formation of Bacillus subtilis. Proc. Natl Acad. Sci. USA 115, 3237-3242 (2018).

23. Chai, L. et al. Isolation, characterization, and aggregation of a structured bacterial matrix precursor. J. Biol. Chem. 288, 17559-17568 (2013).

24. Zakeri, B. et al. Peptide tag forming a rapid covalent bond to a protein, through engineering a bacterial adhesin. Proc. Natl Acad. Sci. USA 109 E690-E697 (2012)

25. Yoshida, S. et al. A bacterium that degrades and assimilates poly(ethylene terephthalate). Science 351, 1196-1199 (2016).

26. Botyanszki, Z., Tay, P. K., Nguyen, P. Q., Nussbaumer, M. G. \& Joshi, N. S. Engineered catalytic biofilms: site-specific enzyme immobilization onto E. coli curli nanofibers. Biotechnol. Bioeng. 112, 2016-2024 (2015).

27. Slavin, Y. N., Asnis, J., Häfeli, U. O. \& Bach, H. Metal nanoparticles: understanding the mechanisms behind antibacterial activity. $J$. Nanobiotechnology 15, 65 (2017)

28. Schaffner, M., Ruhs, P. A., Coulter, F., Kilcher, S. \& Studart, A. R. 3D printing of bacteria into functional complex materials. Sci. Adv. 3 eaao6804 (2017)

29. Grumbein, S. et al. Hydrophobic properties of biofilm-enriched hybrid mortar. Adv. Mater. 28, 8138-8143 (2016).

30. Lieleg, O., Caldara, M., Baumgärtel, R. \& Ribbeck, K. Mechanical robustness of Pseudomonas aeruginosa biofilms. Soft Matter 7, 3307-3314 (2011).

31. Luo, Y., Lode, A. \& Gelinsky, M. Direct plotting of three-dimensional hollow fiber scaffolds based on concentrated alginate pastes for tissue engineering. Adv. Healthc. Mater. 2, 777-783 (2013).
32. Connell, J. L., Ritschdorff, E. T., Whiteley, M. \& Shear, J. B. 3D printing of microscopic bacterial communities. Proc. Natl Acad. Sci. USA 110, 18380-18385 (2013).

33. Orive, G., Tam, S. K., Pedraz, J. L. \& Hallé, J. P. Biocompatibility of alginate-poly-L-lysine microcapsules for cell therapy. Biomaterials. 27, 3691-3700 (2006)

34. Xue, S. et al. A synthetic-biology-inspired therapeutic strategy for targeting and treating hepatogenous diabetes. Mol. Ther. 25, 443-455 (2017).

35. Wilking, J. N., Angelini, T. E., Seminara, A., Brenner, M. P. \& Weitz, D. A. Biofilms as complex fluids. MRS Bull. 36, 385-391 (2011).

36. Flemming, H. C. et al. Biofilms: an emergent form of bacterial life. Nat. Rev. Microbiol. 14, 563-575 (2016)

37. Hochuli, E., Bannwarth, W., Dobeli, H., Gentz, R. \& Stuber, D. Genetic approach to facilitate purification of recombinant proteins with a novel metal chelate adsorbent. Nat. Biotechnol. 6, 1321-1325 (1988).

38. Zhong, C. et al. Strong underwater adhesives made by self-assembling multi-protein nanofibres. Nat. Nanotechnol. 9, 858-866 (2014).

39. Arakaki, A., Webb, J. \& Matsunaga, T. A novel protein tightly bound to bacterial magnetic particles in Magnetospirillum magneticum strain AMB-1. J. Biol. Chem. 278, 8745-8750 (2003).

40. Shaner, N. C. et al. Improved monomeric red, orange and yellow fluorescent proteins derived from Discosoma sp. red fluorescent protein. Nat. Biotechnol. 22, 1567-1572 (2004).

41. McEvoy, A. L. et al. mMaple: a photoconvertible fluorescent protein for use in multiple imaging modalities. PLoS ONE. 7, e51314 (2012).

42. Lu, H. D., Wheeldon, I. R. \& Banta, S. Catalytic biomaterials: engineering organophosphate hydrolase to form self-assembling enzymatic hydrogels. Protein Eng. Des. Sel. 23, 559-566 (2010).

\section{Acknowledgements}

The authors thank D. Kearns (Indiana University) for the kind gift of the B. subtilis 2569 strain, and Y. Liu for the software instruction. Regular TEM characterization was performed at the National Center for Protein Science, Shanghai. Fluorescence microscopy was performed at the Molecular Imaging Core Facility of SLST, Shanghai Tech University. This work was funded by the Science and Technology Commission of Shanghai Municipality (17JC1403900), National Natural Science Foundation of China (No. 31570972), and 2016 Open Financial Fund of Qingdao National Laboratory for Marine Science and Technology (Grant No. QNLM2016ORP0403) for C. Zhong; C.Zhong. also acknowledges start-up funding support from ShanghaiTech University and 1000 Youth Talents Program, granted by the Chinese Central Government. The work was also partially funded by the National Natural Science Foundation of China (No.31872728) for J.H., and the National Natural Science Foundation of China (NSFC: No. 31522017, No. 31470834, No. 31670869) for H.Y

\section{Author contributions}

C. Zhong directed the research; J.H. and C. Zhong conceived of the idea and designed the research. J.H., S.L. and C. Zhang did the rheology and instron measurements. S.L., J.H., J.P., and X.W. assayed the MO and PNP degradation. S.L., J.H., F.B. and Y.W. performed the paraoxon degradation, fluorescence experiments and 3D printing. J.H., C. Zhang, T.Z., and K.L. carried out the molecular constructions. J.H., S.L., and J.Z. performed the HPLC assay, S.X., H.Y., and J.H. did the microencapsulation. J.H., S.L., C. Zhang, and C. Zhong analyzed the data, discussed results, and wrote the manuscript, with the help from LW, C.F. and T.K.L.

\section{Competing interests}

The authors have filed two provisional patents based on this work (CN/201611156490.X and PCT/CN2018/100538). T.K.L. is a co-founder of Senti Biosciences, Synlogic, Engine Biosciences, TangoTherapeutics, Corvium, BiomX, and Eligo Biosciences. T.K.L. also holds financial interests in nest.bio, Ampliphi,IndieBio, and MedicusTek.

\section{Additional information}

Supplementary information is available for this paper at https://doi.org/10.1038/ s41589-018-0169-2.

Reprints and permissions information is available at www.nature.com/reprints.

Correspondence and requests for materials should be addressed to C. Zhong.

Publisher's note: Springer Nature remains neutral with regard to jurisdictional claims in published maps and institutional affiliations.

(C) The Author(s), under exclusive licence to Springer Nature America, Inc. 2018 


\section{Methods}

General methods. Antibiotics for B. subtilis culture were used at the following working concentrations: MLS (erythromycin $(1 \mu \mathrm{g} / \mathrm{mL})$ and lincomycin $(25 \mu \mathrm{g} / \mathrm{mL})$ ); erythromycin $(5 \mu \mathrm{g} / \mathrm{mL})$; spectinomycin $(100 \mu \mathrm{g} / \mathrm{mL})$; chloramphenicol $(5 \mu \mathrm{g} / \mathrm{mL})$. Antibiotics for E. coli culture: carbenicillin $(50 \mu \mathrm{g} / \mathrm{mL})$. The original wild-type strain, B. subtilis 2569, was a generous gift from Dr. Kearns' research group at Indiana University. Detailed information about the strain can be found in a previous publication ${ }^{43}$. A mutant strain with both the tas $A$ and $\sin R$ genes simultaneously knocked out (referred to as $\Delta \operatorname{tas} A \Delta \sin R$ ) and a mutant strain with the $\operatorname{tas} A, \sin R$, and $e p s A \sim O$ genes knocked out (referred to as $\Delta \operatorname{tas} A \Delta \operatorname{sinR} \Delta$ eps) were constructed and sequenced. The original peptide insert regions were either fully synthesized (Genewiz) or PCR-generated. All cloning was performed following standard protocols of molecular cloning or isothermal Gibson Assembly and verified by DNA sequencing (Genewiz). All strains and plasmids used in this study are listed in Supplementary Table 1.

Strain construction. Bacillus subtilis $2569 \Delta \operatorname{tas} A \Delta \operatorname{sinR}$ and Bacillus subtilis 2569 $\Delta \operatorname{tas} A \Delta \sin R \Delta$ eps mutants. To create biofilm-defective strains, we first constructed two suicide plasmids, pMAD- $\Delta$ tasAsinR and pMAD- $\Delta$ eps. Primer pairs of sal-dAF/dcAR were used to amplify the $1-\mathrm{kb}$ fragment from the genome of the wild-type strain upstream of the tas AsinR gene ( $\sin R$ and $\operatorname{tas} A$ genes are next to each other). Primer pairs of daCF/bgl-dCR were used to amplify the $1 \mathrm{~kb}$ fragment downstream of the tasAsin $R$ gene. The two fragments were fused together into a 2 -kb fragment (resulting in deletion of the target $\operatorname{tas} A \sin R$ gene), which was then inserted into pMAD by SalI/BglII digestion to obtain the suicide plasmid pMAD- $\Delta$ $\operatorname{tas} \mathrm{A} \Delta \sin \mathrm{R}$.

Similarly, primer pairs of Bam-d EPS A up F/d EPS A up R, and d EPS O dw F/Sal-d EPS O dw R were used to amplify the 1-kb fragment upstream and downstream of the epsA O gene cluster, respectively. The two fragments were then fused together into a $2 \mathrm{~kb}$ fragment (resulting in deletion of the target epsA O gene cluster), which was then inserted into the pMAD at the BamHI/SalI site to obtain the suicide plasmid pMAD- $\Delta$ eps. The primers used in strain construction are listed in Supplementary Table 3.

The suicide plasmids were then transformed into B. subtilis competent cells, replacing the BamHI/SalI site to free this antibiotic selection marker as previously described ${ }^{44}$. B. subtilis 2569 strain transformed with suicide plasmids was selected on an LB erythromycin agar plate at $30^{\circ} \mathrm{C}$. Transformants were restreaked onto nonselective $\mathrm{LB}$ and grown overnight at $42^{\circ} \mathrm{C}$. Isolates were patched onto $\mathrm{LB}$ agar supplemented with erythromycin at $5 \mu \mathrm{g} / \mathrm{mL}$. PCR fragments from the genomic DNA of mutants were sequenced to confirm the deletions of the tasAsinR gene and the epsA O gene cluster (Supplementary Figs. 18 and 19). The resultant mutant strains used in this study were referred to as B. subtilis $2569 \Delta \operatorname{tas} A \Delta \operatorname{sinR}(\Delta \operatorname{tas} A \Delta$ $\operatorname{sinR}$ ) and B. subtilis $2569 \Delta \operatorname{tas} A \Delta \sin R \Delta$ eps ( $\Delta \operatorname{tas} A \Delta \sin R \Delta$ eps), respectively.

Plasmid construction. The plasmids used in this study were constructed with standard molecular cloning techniques using Gibson assembly kit or restriction endonucleases with T4 DNA ligase. A Bio-Rad S1000 Thermal Cycler with Dual 48/48 Fast Reaction Modules (Bio-Rad) was used to perform PCRs, ligations, and restriction digests. Gel extractions were carried out with QIAquick Gel Extraction Kits (Qiagen). Custom oligonucleotide primers were ordered from Genewiz.

All ligations for plasmid construction were transformed into E. coli strain DH5 $\alpha$ PRO with standard protocols. Isolated colonies were inoculated into LB medium (Fisher) supplemented with $50 \mu \mathrm{g} / \mathrm{ml}$ carbenicillin. Plasmids were extracted with Qiagen QIAprep Spin Miniprep Kits. The constructed plasmids were verified by restriction digest, and corresponding sequences were confirmed by gene sequencing (Genewiz). The parts that constitute the plasmids used in this work are described in Supplementary Table 2.

Plasmids for gene expression containing the grac promoter were constructed by using a pHT01 empty plasmid as a starting point. Primers for tas $A$ were designed based on the $B$. subtilis 168 genome sequence, and a tas $A$ fragment was amplified from the B. subtilis 2569 genome template. Genes for fusion protein $\mathrm{R}$ were synthesized by Genewiz and optimized for $B$. subtilis host expression. The tas $A$ and $R$ genes were connected with linker gene fragments corresponding to GGGSGGGS or GGGGSGGGGS during gene synthesis or PCR processes. Those tas $A-R$ gene fragments were then inserted into the pHT01 plasmid at BamHI/SmaI sites or single SmaI site. The constructed plasmids were named as $\mathrm{pHT} 01-$ tasA-R vectors in this study. All the primers used were listed as Supplementary Table 3. Plasmid maps are described in Supplementary Fig. 20. Gene sequences and amino acid sequences of TasA and fusion proteins are presented in Supplementary Tables 4 and 5 , respectively.

Biofilm culture conditions. Solution-based biofilm culture. Strains were inoculated from frozen glycerol stocks and grown in LB medium supplemented with $5 \mu \mathrm{g} / \mathrm{mL}$ chloramphenicol. Seed cultures were first grown in $5 \mathrm{ml} \mathrm{LB}$ medium (1\% tryptone (Difco), $0.5 \%$ yeast extract (Difco), and $1 \% \mathrm{NaCl}$ ) overnight at $37^{\circ} \mathrm{C}$. The cell pellets collected from the initial seed cultures were then grown for $2-3 \mathrm{~d}$ at $30^{\circ} \mathrm{C}$ in MSgg medium ${ }^{45,46}(\mathrm{pH}=7.0,5 \mathrm{mM}$ potassium phosphate, $100 \mathrm{mM}$ morpholino propanesulfonic acid (MOPS), $2 \mathrm{mM} \mathrm{MgCl}_{2}, 700 \mu \mathrm{M} \mathrm{CaCl}_{2}, 50 \mu \mathrm{M} \mathrm{MnCl}_{2}, 50 \mu \mathrm{M}$ $\mathrm{FeCl}_{3}, 1 \mu \mathrm{M} \mathrm{ZnCl}, 2 \mu \mathrm{M}$ thiamine, $0.5 \%$ glycerol, $0.5 \%$ glutamate, $50 \mu \mathrm{g} / \mathrm{ml}$ tryptophan, and $50 \mu \mathrm{g} / \mathrm{ml}$ phenylalanine) at an initial cell density of $5 \times 10^{7}$ cells $/ \mathrm{mL}$, supplemented with $5 \mu \mathrm{g} / \mathrm{mL}$ chloramphenicol and $0.5 \mathrm{mM}$ IPTG when necessary.

Solid plate-based biofilm culture. For biofilm formation on agar plates, MSgg medium was solidified by the addition of $1.5 \% \mathrm{w} / \mathrm{v}$ Bacto agar (Difco). Similarly to solution-based biofilm culture, seed cultures were first grown overnight at $37^{\circ} \mathrm{C}$. The collected pellets from the seed cultures were then resuspended in MSgg medium supplemented with $5 \mu \mathrm{g} / \mathrm{mL}$ chloramphenicol and $0.5 \mathrm{mM}$ IPTG at an initial cell density of $5 \times 10^{7}$ cells $/ \mathrm{mL}$. 5-10 $\mu \mathrm{L}$ of the above cell solution was then placed on solid MSgg plates supplemented with $5 \mu \mathrm{g} / \mathrm{mL}$ chloramphenicol and $0.5 \mathrm{mM}$ IPTG and then grown at $30^{\circ} \mathrm{C}$ for $2-3 \mathrm{~d}$.

Paraoxon degradation through enzyme/AuNPs hybrid biocatalysis system. The degradation of paraoxon followed two steps of catalysis reactions, enabled by biofilms containing TasA-OPH nanofibers and TasA-HisTag nanofibersimmobilized nickel nitrilotriacetic acid (Ni-NTA) decorated gold nanoparticles (abbreviated as AuNPs).

The full wavelength scanning for the PAR, PNP and PAP was done with a Cary 5000 UV-Vis-NIR Spectrophotometer. The maximum absorption wavelengths for PAR, PNP, and PAP were determined at 290, 405, and $230 \mathrm{~nm}$, respectively, by UV spectra (Supplementary Fig. 7a). The three compounds, PAR, PNP, and $\mathrm{PAP}$, in the co-cultured system were assayed by reverse phase high-performance liquid chromatography (RP-HPLC), and the HPLC standard curves for PNP, PAR, and PAP were shown in Supplementary Fig. $7 \mathrm{~g}-\mathrm{i}$. The reaction kinetics of $\mathrm{OPH}$ catalysis and paranitrophenol reduction is described in Supplementary Notes 1 and 2 , respectively.

(1) Degradation of PAR into PNP with biofilms containing TasA-OPH nanofibers. Biofilms containing TasA-OPH nanofibers from liquid culture were collected by centrifugation at $5,000 \mathrm{~g}$ for $10 \mathrm{~min}$ and resuspended in CHES buffer (2-( $N$-cyclohexylamino) ethane sulfonic acid, Sigma C2885, pH 7.5), supplemented with $50 \mu \mathrm{M} \mathrm{CoCl}_{2}$ (final $\mathrm{OD}_{600}=1$ ). After $1 \mathrm{~h}$ incubation at $30^{\circ} \mathrm{C}$, the pellets were harvested by centrifugation $(5,000 \mathrm{~g} \times 10 \mathrm{~min})$ and resuspended in CHES buffer (final $\mathrm{OD}_{600}=4, \mathrm{pH} 10.0$ ). For each assay, $900 \mu \mathrm{L}$ of test sample was mixed with $100 \mu \mathrm{L}$ of $10 \mathrm{mM}$ paraoxon in $10 \%$ methanol. The reaction mixtures were incubated at $37^{\circ} \mathrm{C}$ for $2 \mathrm{~h}$. Samples were taken every $10 \mathrm{~min}$ to monitor the absorbance of paranitrophenol from the hydrolysis of substrate (paraoxon) at $405 \mathrm{~nm}$ using the Biotek Cytation 5 imaging reader.

(2) PNP reduction based on functional assessment for TasA-HisTag nanofibersanchored AuNPs biofilms. The $\Delta \operatorname{tas} A \Delta \operatorname{sinR} \Delta$ eps/TasA-HisTag strain was inoculated from frozen glycerol stocks and grown in LB medium using $5 \mu \mathrm{g} / \mathrm{ml}$ chloramphenicol. Seed cultures were grown for $12 \mathrm{~h}$ at $37^{\circ} \mathrm{C}$ with shaking at 220 r.p.m. Biofilm cultures were grown at $30^{\circ} \mathrm{C}$ in MSgg medium supplemented with $5 \mu \mathrm{g} / \mathrm{mL}$ chloramphenicol, $0.5 \mathrm{mM}$ IPTG and $100 \mu \mathrm{L}$ $5 \mathrm{nM}$ Ni-NTA-AuNPs in a 12-well plate. After $2 \mathrm{~d}$ of cultivation, biofilms were harvested and resuspended in PBS buffer $\left(\mathrm{OD}_{600}=1\right)$ after washing with $10 \mathrm{~mL}$ PBS buffer five times. The reaction solution was prepared by mixing $4 \mathrm{~mL}$ of $1 \mathrm{mM}$ PNP, $100 \mu \mathrm{L}$ of $2 \mathrm{M} \mathrm{NaBH}_{4}$, and $900 \mu \mathrm{L}$ of the PBS buffer containing TasA-HisTag biofilms. The PNP concentration over reaction time was monitored based on the absorbance peak at $405 \mathrm{~nm}$ using the Biotek Cytation 5 imaging reader. Samples without addition of IPTG or without addition of Ni-NTA-AuNPs (abbreviated as AuNPs) were used as control groups.

(3) Biocatalytic cascade degradation of paraoxon through independently cultured biofilms. $2 \mathrm{~mL}$ of $10 \mathrm{mM}$ paraoxon in $10 \%$ methanol was added to $18 \mathrm{~mL}$ $0.1 \mathrm{M}$ CHES buffer solution containing TasA-OPH biofilms $\left(\mathrm{OD}_{600}=4.0\right.$, $\mathrm{pH} 10.0$ ), and $50 \mu \mathrm{M} \mathrm{CoCl}_{2}$ solution and incubated at $37^{\circ} \mathrm{C}$. Samples were taken every $20 \mathrm{~min}$ to monitor the absorbance of paranitrophenol from the hydrolysis of substrate (paraoxon) at $405 \mathrm{~nm}$ using the Biotek Cytation 5 imaging reader. After $2 \mathrm{~h}, 10 \mathrm{~mL}$ samples were centrifuged to collect the supernatant.After 2-d cultivation, biofilms containing TasA-HisTag nanofibers were harvested and washed with copious amounts of PBS buffer five times and then resuspended in PBS buffer $\left(\mathrm{OD}_{600}=1\right)$. The reaction solution was prepared by adding $8 \mathrm{~mL}$ of the supernatant (collected from step 1 ), $1 \mathrm{~mL}$ of $2 \mathrm{M} \mathrm{NaBH}_{4}$, and $1 \mathrm{~mL}$ of the PBS buffer containing TasA-HisTag biofilms. The reaction mixtures were incubated at room temperature. Samples were taken every $20 \mathrm{~min}$, and PNP degradation was monitored with the absorbance peak at $405 \mathrm{~nm}$ using the Biotek Cytation 5 imaging reader.

(4) Biocatalytic cascade degradation of paraoxon through co-cultured biofilm systems. $\Delta \operatorname{tas} \mathrm{A} \Delta \sin \mathrm{R} \Delta$ eps/TasA-OPH and $\Delta \operatorname{tas} \mathrm{A} \Delta \operatorname{sinR} \Delta$ eps/TasA-HisTag strains were inoculated from frozen glycerol stocks and grown in $10 \mathrm{ml} \mathrm{LB}$ medium with chloramphenicol $(5 \mathrm{\mu g} / \mathrm{ml})$ overnight at $37^{\circ} \mathrm{C}$, separately. The cell pellets collected from the initial seed cultures were then grown for $2 \mathrm{~d}$ at $30^{\circ} \mathrm{C}$ in $50 \mathrm{~mL}$ MSgg medium supplemented with $5 \mu \mathrm{g} / \mathrm{mL}$ chloramphenicol, $0.5 \mathrm{mM}$ IPTG, and $5 \mathrm{nM}$ AuNPs. The biofilms were then harvested and resuspended in CHES buffer ( $\mathrm{pH} 7.5)$ supplemented with $50 \mu \mathrm{M} \mathrm{CoCl}_{2}\left(\mathrm{OD}_{600}=1\right)$. After $1 \mathrm{~h}$ incubation at $30^{\circ} \mathrm{C}$, the pellets were harvested and resuspended into CHES buffer $\left(\mathrm{OD}_{600}=4, \mathrm{pH} 10.0\right) .2 \mathrm{~mL}$ of $10 \mathrm{mM}$ paraoxon in $10 \%$ methanol was mixed with $18 \mathrm{~mL}$ of the hybrid biofilm sample and incubated at $37^{\circ} \mathrm{C}$. Samples were taken every $20 \mathrm{~min}$ to monitor the absorbance of 
paranitrophenol at $405 \mathrm{~nm}$ using the Biotek Cytation 5 imaging reader. After $2 \mathrm{~h}, 1 \mathrm{~mL}$ of $2 \mathrm{M} \mathrm{NaBH}_{4}$ was added to $9 \mathrm{~mL}$ of the above samples, and reaction mixtures were then incubated at room temperature $\left(23 \sim 27^{\circ} \mathrm{C}\right)$. Samples were taken out every $20 \mathrm{~min}$, and the PNP concentration was monitored based on the characteristic absorbance peak at $405 \mathrm{~nm}$ using the Biotek Cytation 5 imaging reader.

MHET degradation with engineered biofilms. Biofilms containing TasA-MHETase nanofibers from liquid culture were collected and resuspended in PBS $\left(\mathrm{OD}_{600}=1\right)$, and then incubated with $400 \mu \mathrm{M}$ of MHET in $40 \mathrm{mM} \mathrm{Na}_{2} \mathrm{HPO}_{4}-\mathrm{HCl}(\mathrm{pH} 7.0)$, $80 \mathrm{mM} \mathrm{NaCl}$, at $30^{\circ} \mathrm{C}$ for $18 \mathrm{~h}$. The reaction was stopped by adding an equal amount of $160 \mathrm{mM}$ phosphate buffer ( $\mathrm{pH} 2.5)$ containing 20\% (v/v) DMSO. The MHET and its degradation product, TPA, were analyzed with RP-HPLC as described above.

\section{Biofabrication of engineered biofilms using 3D printing.}

(1) 3D printing of biofilms. 3 -d cultured biofilms containing TasA-HisTag nanofibers were scraped off from the MSgg plates and placed into the charging barrels of a 3D printer. 3D structures of variable shapes were printed using a robotic 3D printer (GeSim BioScaffolder 3.1). The shapes of polygons or circles were directly printed using the existing graph program in the system, whereas other shapes (for example, the letters representative of ShanghaiTech University) were printed using the imported stl graph program. The printing parameters applied in the experiments were: printing pressure (150-250 kPa), the inner diameter of the nozzle $(160 \mu \mathrm{m})$, and printing speed $(5-10 \mathrm{~mm} / \mathrm{s})$.

(2) 3D printing of biofilms/QDs hybrid structures. Biofilms containing TasAHisTag nanofibers were cultured on MSgg solid plates to which blue (CdZnS@ZnS), red (CdSeS@ZnS), and green (CdZnSeS@ZnS) QDs had been added. The as-grown biofilms with QDs were scraped off from the plates and placed into the 5 - $\mathrm{mL}$ syringe barrels with a nozzle diameter of $160 \mu \mathrm{m}$. 3D structures were fabricated using a three-axis robotic deposition stage (GeSim BioScaffolder 3.1). The 3D shapes of polygons or circles were printed directly using the existing graph program in the system, and other graphics or letters were printed using the imported stl program. The printing parameters applied in the experiments were: printing pressure $(150-250 \mathrm{kPa})$, the inner diameter of the nozzle $(160 \mu \mathrm{m})$, and printing speed $(5-10 \mathrm{~mm} / \mathrm{s})$.

(3) Encapsulation of printed biofilms inside hydrogels through sequential printing. $5 \%(\mathrm{w} / \mathrm{v})$ gelatin and $2 \%(\mathrm{w} / \mathrm{v})$ sodium alginate were first dissolved in MSgg liquid and the viscous solution was then loaded in the barrels. The gelatin/ alginate mixtures were first printed on PVC plates using a 3D printer (GeSim BioScaffolder 3.1), applied with the following printing parameters: printing pressure $(200-300 \mathrm{kPa})$, printing speed $(5-10 \mathrm{~mm} / \mathrm{s})$, and the nozzle diameter $(210 \mu \mathrm{m})$. The $\Delta \operatorname{tas} \mathrm{A} \Delta \operatorname{sinR} \Delta \mathrm{eps} / \mathrm{Tas} \mathrm{A}$-mCherry biofilms were scraped off from the culture plates and placed into the charging barrels. Biofilms were directly printed on top of the printed gelatin/alginate hydrogel layers using the parameters below: printing pressure (150-250 kPa), printing speed $(5-10 \mathrm{~mm} / \mathrm{s})$ and the nozzle diameter $(160 \mu \mathrm{m})$. Afterward, another hydrogel layer composed of $5 \%(\mathrm{w} / \mathrm{v})$ gelatin and $2 \%(\mathrm{w} / \mathrm{v})$ sodium alginate was printed directly on top of the printed biofilms to encapsulate the biofilms inside hydrogels. To solidify the printed structures, the printed samples above were immersed in $500 \mathrm{mM} \mathrm{CaCl}_{2}$ until the sodium alginate hydrogels turned white. The 3D hydrogels were then cultured in MSgg culture media and imaged with stereoscopic microscope Nikon SMZ25 for viability and functional performance evaluations.

Biofabrication of engineered biofilms via microencapsulation. TasA-mCherry nanofibers were first cultured and harvested following the solid-plate biofilm culture protocol described above. The as-prepared biofilms were thoroughly mixed with $1.5 \%$ sodium alginate solution ( $1.5 \%$ low viscosity alginate in morpholino propanesulfonic acid, MOPS washing-buffer). A $25-\mathrm{mL}$ syringe loaded with $20 \mathrm{~mL}$ mixture attached to a reaction vessel was then connected to a control unit of the B-395 Pro encapsulator (BÜCHI Labortechnik AG, Flawil, Switzerland). Alginate micro-gel beads ( $400 \mu \mathrm{m}$ diameters) were fabricated at a flow rate of $20 \mathrm{~mL} / \mathrm{min}$ using a $200-\mu \mathrm{m}$ nozzle, operated at vibration frequency of $1,300 \mathrm{~Hz}$ and voltage of $1.10 \mathrm{kV}$.

After 5 -min solidification in $500 \mathrm{~mL}$ of curing buffer $\left(100 \mathrm{mM} \mathrm{CaCl}_{2}\right.$ and $10 \mathrm{mM}$ MOPS), the micro-gel beads $(10 \mathrm{~mL})$ were sequentially immersed in $75 \mathrm{~mL}$ of $0.05 \%$ poly-L-lysine (PLL) solution $(0.05 \% \mathrm{w} / \mathrm{v}$ poly-L-lysine $(\mathrm{Mw}=15,000$ $\sim 30,000)$ in MOPS washing buffer) and $75 \mathrm{~mL}$ of $0.03 \% \mathrm{w} / \mathrm{v}$ alginate solution $(0.03 \% \mathrm{w} / \mathrm{v}$ low viscosity alginate in MOPS washing-buffer) to form two alternatelayer coated alginate beads, referred to as alginate-poly-(L-lysine)-alginate hydrogel microcapsules. The prepared microcapsules were then immersed in $75 \mathrm{~mL}$ of depolymerization solution ( $50 \mathrm{mM}$ sodium citrate, $0.45 \% \mathrm{NaCl}, 10 \mathrm{mM} \mathrm{MOPS}$ ) for 5-10 min to dissolve the core of the gels.

The fabricated microbeads were finally cultured in MSgg media supplemented with $0.5 \mathrm{mM}$ IPTG inducer. Fluorescent imaging of the microbeads was performed using inverted fluorescence microscopy (Nikon ECLIPSE Ti). The size of the fabricated micro-gel beads is larger than $200 \mu \mathrm{m}$ owing to swelling in solution.
Self-regeneration experiments. Biofilms containing TasA-HisTag nanofibers grown on solid MSgg plates (supplemented with $5 \mu \mathrm{g} / \mathrm{mL}$ chloramphenicol and $0.5 \mathrm{mM}$ IPTG) were scraped off and placed into the charging barrels of a $3 \mathrm{D}$ printer. The biofilms were first printed into designed hexagonal patterns on a piece of glass slide. The edge lengths of the three printed hexagons were 5, 7, and $9 \mathrm{~mm}$ respectively. The $3 \mathrm{D}$ printed biofilms on the glass slide were then put upside down onto a new solid MSgg plate, and the biofilms were able to regrow in hexagonal patterns after $2 \mathrm{~d}$. The biofilms regrowing in the hexagonal patterns on the first nutrient-rich agar plate were placed upside down on the second nutrient-rich agar plate. Similar regeneration experiments were repeated for another two rounds until biofilms regrowing in the hexagonal patterns on the third agar plate were put upside down on the fourth nutrient-rich agar plate.

\section{Long-term viability and functional performance assessment of hydrogel-} trapped biofilms. The printed hydrogels with trapped cells that express TasAmCherry nanofibers and the microcapsules containing trapped cells that express TasA-mCherry nanofibers were produced following the protocols described above. Both samples were immersed into liquid MSgg medium supplemented with $5 \mu \mathrm{g} / \mathrm{mL}$ chloramphenicol and $0.5 \mathrm{mM}$ IPTG. The sandwiched samples were taken out after different induction times for fluorescent detection. To evaluate the long-term viability and functional performance, the hydrogel structures were stored at $4{ }^{\circ} \mathrm{C}$ for 5 weeks and then immersed into liquid MSgg medium supplemented with $5 \mu \mathrm{g} / \mathrm{mL}$ chloramphenicol and $0.5 \mathrm{mM}$ IPTG. The sandwiched samples were taken out for fluorescence detection. Fluorescence imaging was recorded using a stereoscopic microscope (Nikon SMZ25). Hydrogels immersed in media without IPTG were used as control samples.

Crystal Violet (CV) biofilm assay. Quantification of biofilms was carried out following a Crystal Violet (CV) staining protocol described in previous publications $s^{1,47}$. Briefly, $\Delta \operatorname{tas} A \Delta \operatorname{sinR} \Delta$ eps/tasA-R cells, at an initial seeding density of $5 \times 10^{7}$ cells $/ \mathrm{mL}$, were grown in 24 -well plates for $48 \mathrm{~h}$ at $30^{\circ} \mathrm{C}$ in MSgg supplemented with or without $0.5 \mathrm{mM}$ IPTG. Biofilms containing TasA-R nanofibers were collected by centrifugation at $5,000 \mathrm{~g}$ for $10 \mathrm{~min}$. The collected biofilms were washed in $\mathrm{ddH}_{2} \mathrm{O}$ and then recollected by centrifugation at $5,000 \mathrm{~g}$ for $10 \mathrm{~min}$. The samples were then mixed with $400 \mu \mathrm{L}$ of $0.1 \mathrm{w} / \mathrm{v} \%$ aqueous crystal violet (Sigma) and incubated at room temperature for $10-15 \mathrm{~min}$. The samples were then washed four times by immersing the samples in a tub of $\mathrm{ddH}_{2} \mathrm{O}$ until all visible dye was washed off. The washed samples were then recollected by centrifugation at $5,000 \mathrm{~g}$ for $10 \mathrm{~min}$, added to $400 \mu \mathrm{L}$ of $30 \%$ acetic acid aqueous solution, and incubated for $15 \mathrm{~min}$ at RT to solubilize the added CV. Subsequently, $125 \mu \mathrm{L}$ of the solution was transferred to a 96-well plate. Absorbance at $550 \mathrm{~nm}$ of different solutions was measured, with $30 \%$ acetic acid aqueous solution used as a blank.

Transmission electron microscopy (TEM) and immuno-gold labeling. $20 \mu \mathrm{L}$ biofilm liquid culture was dropped onto carbon-coated TEM grids (Zhongjingkeyi Technology, EM Sciences) for 1-5 min. The grids were washed with $20 \mu \mathrm{L}$ PBS buffer and $20 \mu \mathrm{L}$ water. The excess liquid was blotted off on a Whatman no. 1 filter paper, and the sample was negatively stained with $20 \mu \mathrm{L} 1 \mathrm{w} / \mathrm{v} \%$ uranyl acetate solution. The samples were air then dried and examined in a FEI T12 transmission electron microscope at an accelerating voltage of $120 \mathrm{kV}$. Images were taken with an AMT 2k CCD camera.

To image biofilm samples containing anchored nickel nitrilotriacetic aciddecorated gold nanoparticle (Ni-NTA-AuNP), biofilm samples loaded on TEM grids were first rinsed with a $30 \mu \mathrm{L}$ droplet of $\mathrm{ddH}_{2} \mathrm{O}$ three times. The samples were then combined with $30 \mu \mathrm{L}$ selective binding buffer $(1 \times \mathrm{PBS}$, supplemented with $0.487 \mathrm{M} \mathrm{NaCl}, 80 \mathrm{mM}$ imidazole, and $0.2 \% \mathrm{v} / \mathrm{v}$ Tween 20 ), and placed facedown in a $60-\mu \mathrm{L}$ droplet of selective binding buffer with $10 \mathrm{nM} 5 \mathrm{~nm}$ Ni-NTAAuNP particles (Nanoprobes) to incubate for $90 \mathrm{~min}$. The grid was then washed five times with selective binding buffer, then twice with $1 \times$ PBS and twice with $\mathrm{ddH}_{2} \mathrm{O}$. The thoroughly washed grid was then negatively stained and examined in a FEI T12 transmission electron microscope following the same procedure and parameters described above.

For immunolocalization of TasA proteins, biofilm samples were first placed on nickel grids and then immersed in $20 \mu \mathrm{L}$ blocking buffer (PBS buffer containing $1 \%$ skim milk and $0.1 \%$ Tween 20 ) for $30 \mathrm{~min}$, followed by incubation for $2 \mathrm{~h}$ in a $20-\mu \mathrm{L}$ droplet of blocking buffer with anti-TasA primary antibody (Abmart company, 26827-1) diluted at 1:150 ratio.

Afterward, the samples were rinsed three times in $20 \mu \mathrm{L}$ PBST (PBS buffer containing $0.1 \%$ Tween 20 ) and subsequently transferred to a $20-\mu \mathrm{L}$ droplet of blocking buffer with goat anti-rabbit secondary antibody conjugated to 20-nm gold particles (BBI Solutions, Batch 12213, EM.GAR20) diluted at 1:5,000 ratio, and incubated for $1 \mathrm{~h}$. The grids were then washed with $20 \mu \mathrm{L}$ PBS buffer and $20 \mu$ $\mathrm{L} \mathrm{ddH_{2 }} \mathrm{O}$. The excess liquid was blotted off on a filter paper (Whatman no. 1), and the sample was then negatively stained and examined in a FEI T12 transmission electron microscope following the same parameters described above.

Fluorescence microscopy. The $\Delta \operatorname{tas} A \Delta \operatorname{sinR} \Delta$ eps/TasA-mCherry biofilms and the printed samples containing $\Delta \operatorname{tas} A \Delta \operatorname{sinR} \Delta$ eps/TasA-HisTag biofilms anchored with 
diverse QDs (including blue (CdZnS@ZnS), red (Co-NTA CdSeS@ZnS), and green QDs (CdZnSeS@ZnS)) were collected for fluorescence imaging. The fluorescence imaging of 3D printed hydrogels containing TasA-mCherry biofilms was carried out with a Nikon SMZ25 stereoscopic microscope.

Biofilms containing TasA-mCherry nanofibers from liquid culture were collected by centrifugation at $5,000 \mathrm{~g}$ for $10 \mathrm{~min} .10 \mu \mathrm{L}$ of biofilm solution dripped on a glass slide was then imaged under Nikon confocal microscope A1R. Biofilms containing TasA-SpyTag nanofibers from liquid culture were harvested and resuspended with PBS in a tube. $0.5 \mathrm{~mL} 1 \mathrm{mg} / \mathrm{mL}$ purified SpyCatcher-GFP proteins in PBS was added to tubes, incubated for $1 \mathrm{~h}$ and then washed three times with PBS buffer. $10 \mu \mathrm{L}$ of the mixture solution dripped on a slide was then imaged under Zeiss Axio Imager 2. Fluorescence imaging of the three different samples (TasA-SpyTag biofilm added to SpyCatcher-GFP protein, TasA-SpyTag biofilm added to free GFP protein, and TasA-HisTag biofilm added to SpyCatcher-GFP protein) was carried out following the same protocol described above.

Fluorescence spectra. Fluorescence features of the biofilms/QDs hybrid structures were characterized using a fluorescence spectrometer. $1 \mathrm{~g}$ of the $\Delta \operatorname{tas} A \Delta \operatorname{sinR} \Delta$ eps/TasA-HisTag biofilms was mixed with $200 \mu \mathrm{L}$ QDs (Blue (CdZnS@ZnS), red (CdSeS@ZnS) or green (CdZnSeS@ZnS) QDs) to produce biofilm/QDs hybrid structures. $10 \mathrm{mg}$ of the above samples were then coated on a glass slide. Fluorescence spectra of the coated samples were collected using HORIBA FL-3 at an excitation wavelength of $350 \mathrm{~nm}$.

Reverse phase high-performance liquid chromatography (RP-HPLC) analysis. RP-HPLC was used to monitor PAR and MHET and their associated degradation products. RP-HPLC was performed on an Agilent 1260 Infinity system (Agilent Technologies) equipped with a ZORBAX SB-C18 guard column $(4.6 \times 12.5 \mathrm{~mm}$, $5 \mu \mathrm{m})$ and a ZORBAX SB-C18 analytical column $(4.6 \times 150 \mathrm{~mm}, 5 \mu \mathrm{m})$ from Agilent Technologies. The mobile phase was methanol and $20 \mathrm{mM}$ phosphate buffer ( $\mathrm{pH} 2.5$ ) at a flow rate of $1.0 \mathrm{~mL} / \mathrm{min}$, and the effluent was monitored at a wavelength of $240 \mathrm{~nm}$ with a UV detector. The typical elution conditions applied were: 0 to $15 \mathrm{~min}, 25 \%$ (v/v) methanol; 15 to $25 \mathrm{~min}, 25-95 \%$ methanol linear gradient; 25 to $30 \mathrm{~min}, 95-25 \%$ methanol linear gradient.

Contact angle measurement. Biofilms grown on MSgg agar plates were carefully isolated, and the contact angle of these biofilms was then measured at room temperature using a Theta Lite optical tensiometer (Biolin) following the sessile drop method ${ }^{48}$.

Rheological properties of engineered biofilms. The rheological properties of engineered biofilms were evaluated on a strain-controlled rheometer (Anton paar MCR101) equipped with a $24.948-\mathrm{mm}$ diameter cone-plate ( $48 \mu \mathrm{m}$ gap). All biofilm samples were scraped from MSgg plate and placed on the cone plate. To minimize water evaporation in biofilms, a closed surrounding chamber containing pure water was used. To determine the linear viscoelastic region of the biofilm samples, strain sweep experiments from $0.01 \sim 100 \%$ strain amplitude were performed at a fixed frequency of $1 \mathrm{rad} / \mathrm{s}$. Frequency sweep experiments from 100 to $0.01 \mathrm{rad} / \mathrm{s}$ were performed at $1 \%$ strain amplitude. The temperature was kept at a constant temperature of $25^{\circ} \mathrm{C}$ throughout the experiments with a Peltier thermoelectric device.
Instantaneous recovery capacity of the viscoelastic networks of the WT and TasA-HisTag biofilms was measured by an oscillatory time sweep $(0.1 \%$ amplitude strain, $5 \mathrm{rad} / \mathrm{s}$ angular frequency) for $120 \mathrm{~s}$, followed by a sudden shear process in a steady state $\left(100 \mathrm{~s}^{-1}\right)$ for $120 \mathrm{~s}$ and an oscillatory time sweep $(0.1 \%$ amplitude strain, $5 \mathrm{rad} / \mathrm{s}$ angular frequency) for another $120 \mathrm{~s}\left(\right.$ ref. ${ }^{30}$ ). The data were presented in Supplementary Fig. 10

Quantum dots (QDs) toxicity assay. The biofilm-deficient strain (pHT01), and the TasA and TasA-HisTag biofilm-producing strains were inoculated from frozen glycerol stocks and grown in LB medium with $5 \mu \mathrm{g} / \mathrm{mL}$ chloramphenicol. Seed cultures were grown in LB medium for $12 \mathrm{~h}$ at $37^{\circ} \mathrm{C}$. Static biofilm samples were grown at $30^{\circ} \mathrm{C}$ in MSgg medium containing $5 \mu \mathrm{g} / \mathrm{mL}$ chloramphenicol and 0.5 mM IPTG, either with or without 10\% red Co-NTA CdSeS@ZnS QDs. Biofilm production for different biofilm cultures was measured after 2-d cultivation in the presence or absence of QDs using a standard crystal-violet (CV) assay. Influences of QDs on biofilm growth and production among three different types of biofilms were assessed and compared.

Statistical analysis. All results presented in graphs show the mean data \pm s.e.m. Number of experiments and samples was described in the figure legends. A twosided Student's $t$-test was used to calculate $P$ values. No statistical method was used to predetermine sample size.

Reporting Summary. Further information on research design is available in the Nature Research Reporting Summary linked to this article.

\section{Data availability}

The main data supporting the findings of this study are available within the article and its Supplementary Information files. Extra data are available from the corresponding author upon reasonable request.

\section{References}

43. Konkol, M. A., Blair, K. M. \& Kearns, D. B. Plasmid-encoded ComI inhibits competence in the ancestral 3610 strain of Bacillus subtilis. J. Bacteriol. 195, 4085-4093 (2013)

44. Arnaud, M., Chastanet, A. \& Débarbouillé, M. New vector for efficient allelic replacement in naturally nontransformable, low-GC-content, gram-positive bacteria. Appl. Environ. Microbiol. 70, 6887-6891 (2004)

45. Romero, D., Aguilar, C., Losick, R. \& Kolter, R. Amyloid fibers provide structural integrity to Bacillus subtilis biofilms. Proc. Natl Acad. Sci. USA 107, 2230-2234 (2010)

46. Branda, S. S., González-Pastor, J. E., Ben-Yehuda, S., Losick, R. \& Kolter, R. Fruiting body formation by Bacillus subtilis. Proc. Natl Acad. Sci. USA 98, 11621-11626 (2001)

47. Branda, S. S., Chu, F., Kearns, D. B., Losick, R. \& Kolter, R. A major protein component of the Bacillus subtilis biofilm matrix. Mol. Microbiol. 59, 1229-1238 (2006)

48. Arnaouteli, S. et al. Bifunctionality of a biofilm matrix protein controlled by redox state. Proc. Natl Acad. Sci. USA 114, E6184-E6191 (2017). 


\section{Reporting Summary}

Nature Research wishes to improve the reproducibility of the work that we publish. This form provides structure for consistency and transparency in reporting. For further information on Nature Research policies, see Authors \& Referees and the Editorial Policy Checklist.

\section{Statistical parameters}

When statistical analyses are reported, confirm that the following items are present in the relevant location (e.g. figure legend, table legend, main text, or Methods section).

n/a Confirmed

$\bigotimes$ The exact sample size $(n)$ for each experimental group/condition, given as a discrete number and unit of measurement

$\bigotimes$ An indication of whether measurements were taken from distinct samples or whether the same sample was measured repeatedly

The statistical test(s) used AND whether they are one- or two-sided

Only common tests should be described solely by name; describe more complex techniques in the Methods section.

Х $\square$ A description of all covariates tested

Х $\square$ A description of any assumptions or corrections, such as tests of normality and adjustment for multiple comparisons

$\square \bigotimes$ A full description of the statistics including central tendency (e.g. means) or other basic estimates (e.g. regression coefficient) AND variation (e.g. standard deviation) or associated estimates of uncertainty (e.g. confidence intervals)

For null hypothesis testing, the test statistic (e.g. $F, t, r$ ) with confidence intervals, effect sizes, degrees of freedom and $P$ value noted Give $P$ values as exact values whenever suitable.

Х $\square$ For Bayesian analysis, information on the choice of priors and Markov chain Monte Carlo settings

Х $\square$ For hierarchical and complex designs, identification of the appropriate level for tests and full reporting of outcomes

Х $\square$ Estimates of effect sizes (e.g. Cohen's $d$, Pearson's $r$ ), indicating how they were calculated

Clearly defined error bars

State explicitly what error bars represent (e.g. SD, SE, CI)

Our web collection on statistics for biologists may be useful.

\section{Software and code}

Policy information about availability of computer code

Data collection Three-dimensional printed "sandwiched" biofilm structures were printed using a BioScaffolder3.1 3D printer (GeSim).

The microencapsulation was fabricated using a B-395 Pro encapsulator (BÜCHI Labortechnik AG).

The TEM images of biofilms were taken with an AMT 2k CCD camera.

The degradation of PAR and MHET were monitored using RP-HPLC performed on an Agilent 1260 Infinity system (Agilent Technologies) equipped with a ZORBAX SB-C18 guard column $(4.6 \times 12.5 \mathrm{~mm}, 5 \mu \mathrm{m})$ and a ZORBAX SB-C18 analytical column $(4.6 \times 150 \mathrm{~mm}, 5 \mu \mathrm{m})$ from Agilent Technologies.

The contact angle of biofilms were measured with a Theta Lite optical tensiometer (Biolin).

The rheological properties of engineered biofilms were assessed using a strain-controlled rheometer (Anton paar MCR101) equipped with a $24.948 \mathrm{~mm}$ diameter cone.

The fluorescent imaging was performed using Laser Scanning Confocal Microscopy LSM 710 (Zeiss). The fluorescent imaging of 3D printed hydrogels containing TasA-mCherry biofilms was carried out with a Nikon SMZ25 stereoscopic microscope.

Fluorescence spectra of biofilms/QDs complex structures were collected using a HORIBA FL-3 Spectrofluorometer with excitation of 350 nm light.

Data analysis

All data were plotted using the OriginPro version 9.4.2 for Windows (Origin Software, Northampton, USA).

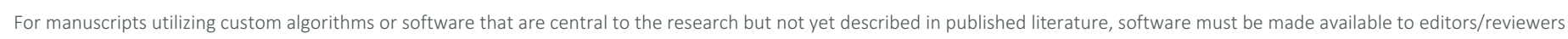

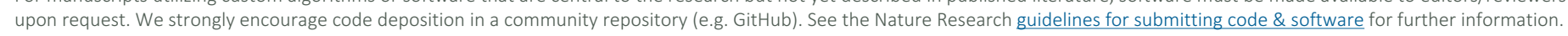


Policy information about availability of data

All manuscripts must include a data availability statement. This statement should provide the following information, where applicable:

- Accession codes, unique identifiers, or web links for publicly available datasets

- A list of figures that have associated raw data

- A description of any restrictions on data availability

The main data supporting the findings of this study are available within the article and its Supplementary Information files. Extra data are available from the corresponding author upon reasonable request.

\section{Field-specific reporting}

Please select the best fit for your research. If you are not sure, read the appropriate sections before making your selection.

$\bigotimes$ Life sciences $\quad \square$ Behavioural \& social sciences $\quad \square$ Ecological, evolutionary \& environmental sciences

For a reference copy of the document with all sections, see nature.com/authors/policies/ReportingSummary-flat.pdf

\section{Life sciences study design}

All studies must disclose on these points even when the disclosure is negative.

Sample size Sample sizes were not predetermined. We generally used sample sizes of 3 technical replicates, which can provide necessary statistical support. We used 100 replicate samples to measure the diameters of three different nanofibres produced in TasA-HisTag, TasA-mefp5, and TasA-OPH biofilms and used 50 replicate samples to measure the diameters of four separate types of biofilms (WT, TasA-HisTag, TasA-mefp5, and TasA-OPH biofilms).

Data exclusions No data were excluded from the analyses.

Replication All attempts at replication were successful. We described details for each experiment in online method to reproduce all our experiments.

Randomization Randomization was not applicable because we measured the exact properties of the biofilm.

Blinding No blinding was conducted, because blinding would not provide any reliable datasets for our all biochemical and genetic experiments.

\section{Reporting for specific materials, systems and methods}

\section{Materials \& experimental systems}

$\mathrm{n} / \mathrm{a}$ Involved in the study

$\square \bigotimes$ Unique biological materials

$\square$ Antibodies

$\triangle \square$ Eukaryotic cell lines

\\ Palaeontology

$\bigotimes \square$ Animals and other organisms

Х $\square$ Human research participants

\section{Unique biological materials}

\section{Policy information about availability of materials}

Obtaining unique materials The original wild-type strain, B. subtilis 2569, was a generous gift from Dr. Kearns' research group at Indiana University. The plasmid pHT01 was a lab collection and will be sent for basic research purposes upon reasonable request.

\section{Antibodies}

Antibodies used

The rabbit anti-TasA primary antibody was synthesized by Abmart company diluted at 1:150 ratio.

\begin{tabular}{l|l}
\multicolumn{2}{l}{ Methods } \\
\hline n/a Involved in the study \\
$\square$ \\
$\square$ ChIP-seq \\
$\square$ Flow cytometry
\end{tabular}

Х $\square$ MRI-based neuroimaging 
The goat anti-rabbit 20-nm gold secondary antibody was purchase from BBI Solutions diluted at 1:5000 ratio. (Batch 12213, EM.GAR20)

The rabbit anti-TasA primary antibody and the goat anti-rabbit 20-nm gold secondary antibody were validated by immuno-gold labeling using transmission electron microscopy. 